\title{
Allotropic Carbon Nanoforms as Advanced Metal-Free Catalysts or as Supports
}

\author{
Hermenegildo Garcia \\ Instituto Universitario de Tecnología Química CSIC-UPV, Universidad Politécnica de Valencia, Avenida De Los Naranjos s/n, \\ 46022 Valencia, Spain
}

Correspondence should be addressed to Hermenegildo Garcia; hgarcia@qim.upv.es

Received 19 May 2014; Revised 14 July 2014; Accepted 28 July 2014; Published 15 September 2014

Academic Editor: Davut Avci

Copyright (C) 2014 Hermenegildo Garcia. This is an open access article distributed under the Creative Commons Attribution License, which permits unrestricted use, distribution, and reproduction in any medium, provided the original work is properly cited.

\begin{abstract}
This perspective paper summarizes the use of three nanostructured carbon allotropes as metal-free catalysts ("carbocatalysts") or as supports of metal nanoparticles. After an introductory section commenting the interest of developing metal-free catalysts and main features of carbon nanoforms, the main body of this paper is focused on exemplifying the opportunities that carbon nanotubes, graphene, and diamond nanoparticles offer to develop advanced catalysts having active sites based on carbon in the absence of transition metals or as large area supports with special morphology and unique properties. The final section provides my personal view on future developments in this field.
\end{abstract}

\section{Introduction: From Active Carbons to Carbon Allotropes}

In classical heterogeneous catalysis, active carbons (ACs) have been widely used as supports for noble metal and metal oxides [1-3]. ACs are high surface area materials having carbon as predominant element in their composition that are obtained by pyrolysis of available biomass wastes, upon addition of inorganic reagents to promote the carbonisation process. For instance, one popular active carbon comes from coconut shells adequately powdered, pyrolyzed at $600^{\circ} \mathrm{C}$ under $\mathrm{N}_{2}$, mixed with phosphoric acid for activation, and then baked at temperatures below $300^{\circ} \mathrm{C}[4,5]$. In other recipes, olive seeds or almond shells are used as AC precursors and other mineral acids or oxidizing chemicals are employed as additives [6-8].

The structure of ACs is poorly defined with domains of amorphous carbon and the presence of condensed polycyclic aromatic compounds forming platelets of nanometric dimensions that are interconnected by bridges that can be $\mathrm{CH}_{2}$ and heteroatoms such as $\mathrm{O}, \mathrm{NH}$, and $\mathrm{S}$. In certain regions, ACs have graphitic domains when the platelets are large enough and stacking of the imperfect graphene $(G)$ sheets can take place. Besides oxygen and other elements such as nitrogen or sulphur, metal traces such as iron, zinc, and copper, are very frequently present in the final composition of the material because these transition metals have been introduced as additives in the pyrolysis process and they remain in residual, sometimes not negligible, percentages.

Understanding the mechanism in heterogeneous catalysis largely depends on the exhaustive characterization of the solid catalyst and on the knowledge on the architecture of the active sites [9]. In this sense, while ACs are available and affordable materials exhibiting high adsorption capacity, this property being suitable for their use as support, they are too complex and ill-defined to allow structural characterization and, moreover, they make impossible the preparation of single-site catalysts.

One of the main problems in heterogeneous catalysis derives from the fact that solids contain a wide distribution of centers in which the architecture of the sites and the surroundings change from one specific site to another and, as result, the activity and selectivity may change from one site to the neighbor. Optimal solid catalysts would require a material in which all the sites are identical ("single site"), all of them exhibiting the same selectivity and, desirably, the 
maximal activity. Design and synthesis of single-site catalysts has been a continued task in heterogeneous catalysis with the long-term aim of the development of solid catalysts with the highest activity and selectivity [10-12].

In the last decades carbon allotropes with nanometric particle dimension have been characterized and have become commercially available. Since their structure is much better defined than ACs, there has been a continued growing interest in the application of these carbon nanoforms in catalysis as a logical evolution of the use of ACs [13-15]. The interest of allotropic carbon nanoforms in catalysis is, therefore, a logical evolution of the continued use of ACs for the preparation of heterogeneous catalysts but goes much beyond ACs, since the point is now to incorporate the active sites on the carbon structure.

As in the case of ACs, the simplest possibility has been the use of these carbon allotropes as large area supports of active sites [16-18], but the most recent research front is to implement the catalytic sites on the carbon allotrope itself in the absence of metals ("carbocatalysis"). Starting from an ideal structure of the carbon allotrope, it should be possible to introduce sites by creating carbon vacancies, carbon with dangling bonds, structural defects due to carbon vacancies, and oxygenated functional groups or by replacing carbon atoms by other elements ("doping").

The term "carbocatalysts" refers to the development of catalysts based exclusively or predominantly on the use of carbon materials, avoiding or minimizing the dependency of catalysis on the use of metals [19-25]. While the use of metals is considered not "sustainable" due to the limited available resources, carbonaceous catalysts, particularly those derived from biomass, are renewable and affordable and considered as "sustainable" materials.

Among the different carbon allotropes that have been applied in heterogeneous catalysis, the initial studies were based on carbon nanotubes (CNTs) because these materials become commercially available earlier than other carbon materials $[26,27]$. Preparation of CNTs requires catalysts and special equipment. CNTs typically are obtained in small batch quantities and impurified with the catalyst used in their synthesis, typically iron or iron-cobalt alloys dispersed in a matrix to maintain the particle size small. This makes necessary in commercial CNTs several steps of purification, oxidation, and other modification processes before they can be used as catalysts. After CNTs, the use of diamond nanoparticles (DNPs) has also become possible. DNPs can be obtained by milling of diamond powders or by explosive detonation [28]. In the last case, D nanocrystals are embedded on a soot matrix of amorphous carbon that has to be removed before the use of the DNPs as supports [29]. More recently, a new line of research has appeared trying to exploit the opportunities of G-based materials in catalysis [20, 21, 30, 31].

Allotropic carbon nanoforms can be dispersed in suspension in a liquid phase as inks that allow the recovery of the carbonaceous materials by filtration or centrifugation after their use. The term "pseudohomogeneous catalysis" has been used to denote the fact that during the reaction there is no apparent phase differentiation between substrates and catalysts, but after the reaction the carbonaceous material

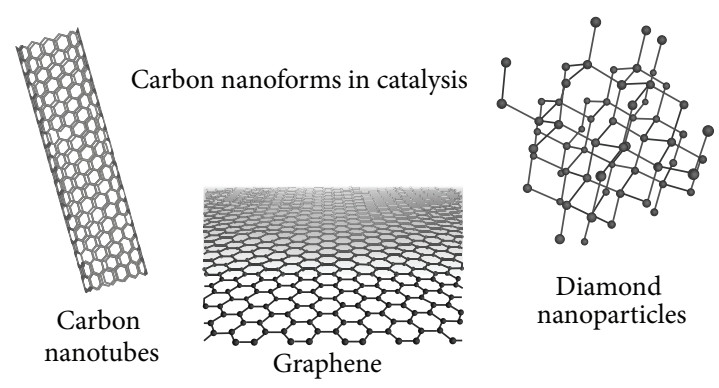

Scheme 1: Structure of the three carbon allotropes whose use as carbocatalysts or as supports of metal nanoparticles will be the topic of the present perspective paper.

can be easily separated and recovered as is characteristic in heterogeneous catalysis [32].

In the present spotlight paper, I will comment on some of the possibilities that nanometric carbon allotropes offer as catalysts or as supports, showing the logic of the evolution from ACs or from other supports to these carbonaceous nanomaterials as catalysts. Scheme 1 contains the type and structure of the carbon nanoforms whose catalytic activity will be commented on in this perspective paper. When possible, comparison between the performance of different carbon allotropes will be made, but I will try to highlight the features of each type of carbon nanoform that make them best suited for certain catalytic applications. I will start with the possibilities and limitations of CNTs, followed by the use of G-based materials in catalysis and finishing with applications of DNPs as supports. In this paper, I will cover a broad range of articles in this area, with emphasis on the use of these materials as carbocatalysts in the absence of metals with the aim of illustrating the broad potential that carbon nanoforms offer in catalysis. In the last section, I will summarize the major points and will provide my view on possible future developments and targets in this area.

\section{Catalysts Based on CNTs as Supports}

CNTs are characterized by their long aspect ratio in which one or several concentric hexagonal arrangements of $\mathrm{sp}^{2}$ carbons ("graphene sheets") form a cylinder with nanometric diameter, but lengths that can reach tens of micrometers. The long aspect ratio and the curvature of the graphene walls are the main characteristics of CNTs.

The synthesis of CNTs, either single wall (SWCNTs) or multiple wall (MWCNTs), is difficult to control and relies on the use of a metal catalyst, typically Fe and Co alloys in the form of small metal NPs, to decompose in the pyrolytic process at temperatures about $500^{\circ} \mathrm{C}$ or above the organic precursor in the absence of oxygen and effect the nucleation and growth of CNTs from elemental carbon atoms generated under these reductive conditions (Scheme 2) [34].

Due to the use of a catalyst in the preparation of CNTs, the amount of CNTs that is available in each batch is limited compared to other carbon nanoforms [36]. MWCNTs can be prepared in larger quantities than SWCNTs because the 


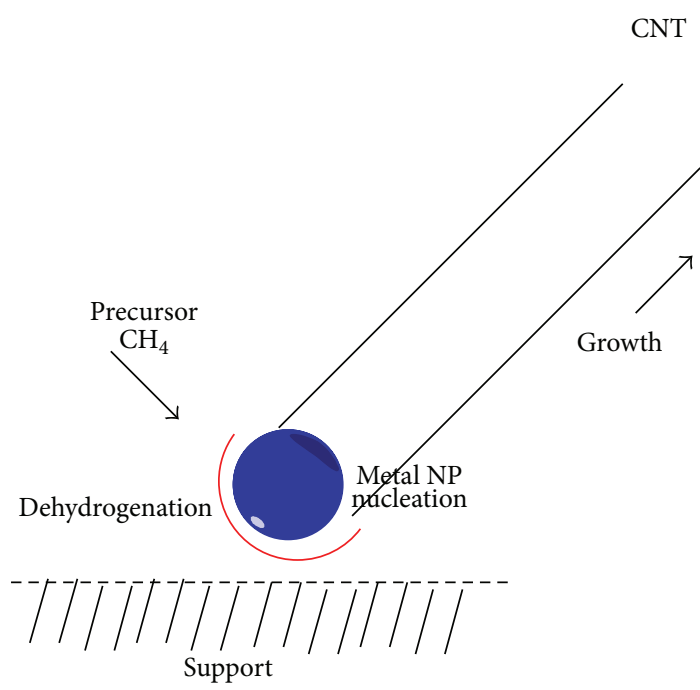

Scheme 2: Pictorial illustration of the synthesis of CNT by dehydrogenative carbonisation of methane on hot metal nanoparticles. On the surface of the metal carbon atoms are continuously being formed at high temperatures due to the dehydrogenative decomposition of the precursors (methane in this case) and these atoms condense in an hexagonal arrangement leading to the graphene wall with diameter commensurate with the dimensions of the metal nanoparticle catalyst and growing outside these nanoparticles.

control on the particle size of the metal alloy is not so strict. In general terms, the advantages of using single wall with respect to multiwall have not been clearly demonstrated in catalysis and, therefore, the use of more stable and affordable MWCNTs can be advantageous with respect to SWCNTs. However, from the point of view of achieving the highest catalytic activity and having well-defined structure, SWCNTs can be preferable since all the carbons are exposed at the surface and their morphology and structure is well defined and can be determined by electron microscopy. Also, from the catalytic point of view, there are no examples showing differences between the properties of conductive or semiconductive SWCNTs as catalysts [37,38]. Electrical conductivity in CNTs depends on the way in which the hexagons of the graphene wall are aligned along the long axis, either spiral (semiconductive) or perpendicular (conductive).

During the purification of CNTs to remove the metal catalyst employed in the synthesis of CNTs, strong inorganic acids are generally needed. $\mathrm{HNO}_{3}$ is one of the preferred reagents for $\mathrm{CNT}$ purification and, then, at the same time that the acid dissolves inorganic impurities, mild oxidation of the graphene wall forming oxygenated functionalities and particularly carboxylic acid groups can also be produced. By performing treatment with $\mathrm{HNO}_{3}$ under harsh conditions of concentration, temperature, and time, purification of the organic residues can be accompanied by cutting of the CNTs from micrometers to hundreds of nanometers $[39,40]$, the cut taking place preferentially by oxidation of the graphene wall at defects. One consequence of the shorter length and formation of oxygenated functional groups is an increase on the dispersability of CNTs in liquid media and water in

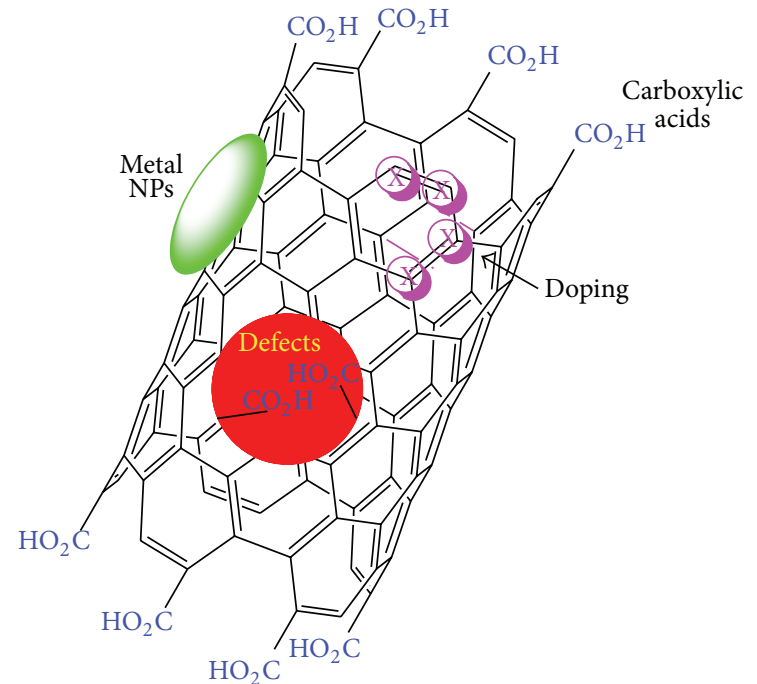

Scheme 3: Possible sites present in CNTs that can exhibit catalytic activity.

particular [41, 42]. Preparation of permanent suspensions of short CNTs is very adequate for the development of pseudohomogeneous catalysis.

CNTs can contain active sites due to the presence of carboxylic acid groups at the rims and wall defects that can be functionalized. Also CNTs can be doped with some heteroatoms, the most common one being $\mathrm{N}$ atoms. Other possible defects include carbon vacancies and doping with some heteroatoms replacing carbon atoms in the wall. CNTs can also be the support of metal NPs that can be the active sites in the reaction. Scheme 3 summarizes some of the possibilities that CNTs offer to incorporate active centers.

However, as commented earlier, the cumbersome preparation and purification of CNTs explain why these carbon nanoforms have not become as widely available and affordable for the catalytic community as desirable. Since it is expected that the activity of these modified CNTs as acid carbocatalysts would be similar to that of more easily available $\mathrm{G}$ materials or that of polymeric resins having carboxylic acids, there is no special reasons beyond morphology and the possibility to encapsulate metal NPs inside the tubes to prefer CNTs in catalysis with respect to G. Thus, although it would be desirable much more information about the catalytic activity of CNTs, it is unlikely that this research front will develop strongly in the near future and, perhaps, the major point of interest will be to compare the catalytic activity of CNTs having curved graphene walls with that of other Gbased catalysts.

CNTs in the absence of any metal have been found to be suitable catalysts for the oxidative dehydrogenation of hydrocarbons (Scheme 4) [43-45]. This process has considerable interest in petrochemical industry for the transformation of propane into propene and butanes into butenes and butadiene. CNTs have defects that typically correspond to oxygenated functional groups, namely, carboxylic acids, quinone-like carbonyl groups, and hydroxyl groups. As I 


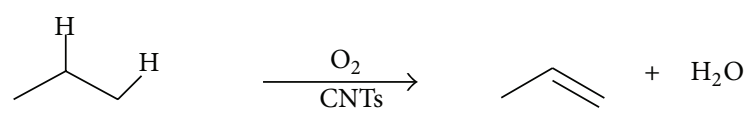

SCHEME 4: Oxidative dehydrogenation of propane catalysed by CNTs.

will comment along this paper, one of the issues that still has to be clarified in many reactions is the nature of the active sites responsible for promoting the reaction. In the present case, using an elegant strategy, the catalytic activity of CNTs for the oxidative dehydrogenation of light alkanes was compared to that of analogous CNTs samples that have been modified to mask selectively each type of the oxygenated functional groups [46]. In this way, carboxylic and hydroxyl groups were selectively protected by esterification or substitution, respectively, while quinone-like carbonyl groups were transformed into imines. Comparison of the catalytic activity of these modified CNTs having selectively masked one of the three possible functional groups has shown that the catalytic activity of pristine CNTs and that of the esterified or hydroxyl substituted CNTs are almost identical and about four times higher than that of the imine functionalized CNTs (Scheme 5). This decrease in the catalytic activity for the modified CNTs that do not contain quinone-like carbonyls but contain carboxylic and hydroxyl groups has led to the conclusion that quinone carbonyls are the functional groups that are responsible for promoting this dehydrogenation [46]. This type of studies shed light on the nature of the catalytically relevant sites and can serve to prepare carbocatalysts with the maximum density of these functional groups and, presumably, with the optimal catalytic activity and selectivity.

Considering that the aim of carbocatalysis is to develop catalysts to replace metals, one challenging reaction that is known to be promoted by transition and noble metals is hydroperoxide decomposition. Using transition metal ions, hydroperoxide decomposition can consume stoichiometric amounts, leading to the formation of considerable amounts of wastes in the reaction. One example of this type of reactions is the Fenton decomposition of $\mathrm{H}_{2} \mathrm{O}_{2}$ by $\mathrm{Fe}^{2+}$ ions in water at acidic $\mathrm{pH}$ values $[47,48]$. Therefore, there is an increasing interest in developing catalytic versions of this hydroperoxide decomposition [47, 48].

In the simplest mechanism, the presence of redox sites that can reversibly donate one electron reducing hydroperoxide and producing the reductive cleavage of the $\mathrm{O}-\mathrm{O}$ bond and then become reoxidized by other hydroperoxide molecules rendering oxygen should catalyze hydroperoxide decomposition. Scheme 6 illustrates the catalytic cycle of hydroperoxide decomposition by the presence of a redox site that really can be considered as hydroperoxide dismutation. Hydroperoxides have oxygen atoms in the $-\mathrm{I}$ oxidation state and can undergo disproportionation to the 0 and -II oxidation states. Transition metals having different oxidation states and binding strongly to hydroperoxides have been the preferred catalyst for this process.
However, carbon materials can also have other types of redox sites, for instance, those based on quinonehydroquinone pairs having adequate redox potential to promote the process. In this context, not surprisingly, it has been reported that CNTs can promote benzene hydroxylation to phenol by $\mathrm{H}_{2} \mathrm{O}_{2}$ with high selectivity [49-51]. The process still requires deeper study and understanding in order to increase the efficiency and, particularly, to assess the nature of the active site, but it is interesting to note that the curvature of the graphene wall in MWCNTs has been invoked as playing a key role in the reaction mechanism [49]. It would be important to check this hypothesis by comparing the process with CNTs of different diameters or even other G-based materials.

In the previous process, the synthesis of a chemical compound, phenol from benzene, is the target of the reaction. However, most frequently peroxide decomposition is used to degrade organic compounds present in aqueous phase. Also MWCNTs have been reported to act as catalysts for this type of reaction. Thus, peroxy monosulfate can decompose by the presence of MWCNTs leading to the formation of sulfate radicals that are able to initiate the aerobic decolorization of methylene blue and decomposition of 2,4-dichlorophenol [52]. For this reaction, active carbons also exhibit catalytic activity, but it has been found that reduced graphene oxide (GO) can exhibit even higher activity than MWCNTs [52].

Doping can be a viable general strategy to introduce active sites in CNTs. As a general observation, the presence of $\mathrm{N}$ increases the stability of the carbon nanoforms against oxidation, and, therefore, $\mathrm{N}$ doping makes CNTs more suitable as oxidation catalysts $[53,54]$. N-doped MWCNTs are also able to promote the aerobic oxidation of benzyl alcohols at moderate temperatures $[55,56]$. These $\mathrm{N}$-doped MWCNTs exhibit for this reaction similar catalytic activity as $\mathrm{G}$ materials.

Although in general similar performance as catalysts should be expected for CNTs and G materials, one peculiarity of CNTs is the possibility to include inside the tube of nanometric dimension some metal nanoparticles (NPs) of size smaller than the diameter of the tubes. In this regard, Fe NPs have been confined inside CNTs and the resulting material used for the aerobic oxidation of cyclohexane to adipic acid, a process of large industrial relevance (Scheme 7) [57]. However, the study has to show conclusively that the system based on the inclusion of Fe NPs on CNTs is stable under the reaction conditions and does not undergo self-degradation during the course of the reaction. Carbon nanoforms can be oxidized under various conditions. This oxidation leads to the formation of oxygenated functional groups resulting in the creation of defects. In the case of CNTs, oxidation can result in a shortening of their length as consequence of the oxidative cutting of the tube. Thus, it is very likely that CNTs could undergo an increasing degree of oxidation that eventually could lead to the release of Fe NPs and the deactivation of the catalyst, but this issue of catalyst stability in aerobic oxidations has not been yet addressed.

Besides oxidation, carbon nanoforms and also CNTs have attracted interest as catalyst for reversible hydrogen release/uptake from metal hydrides [58-61]. In the context of hydrogen storage, one of the possibilities that has been 


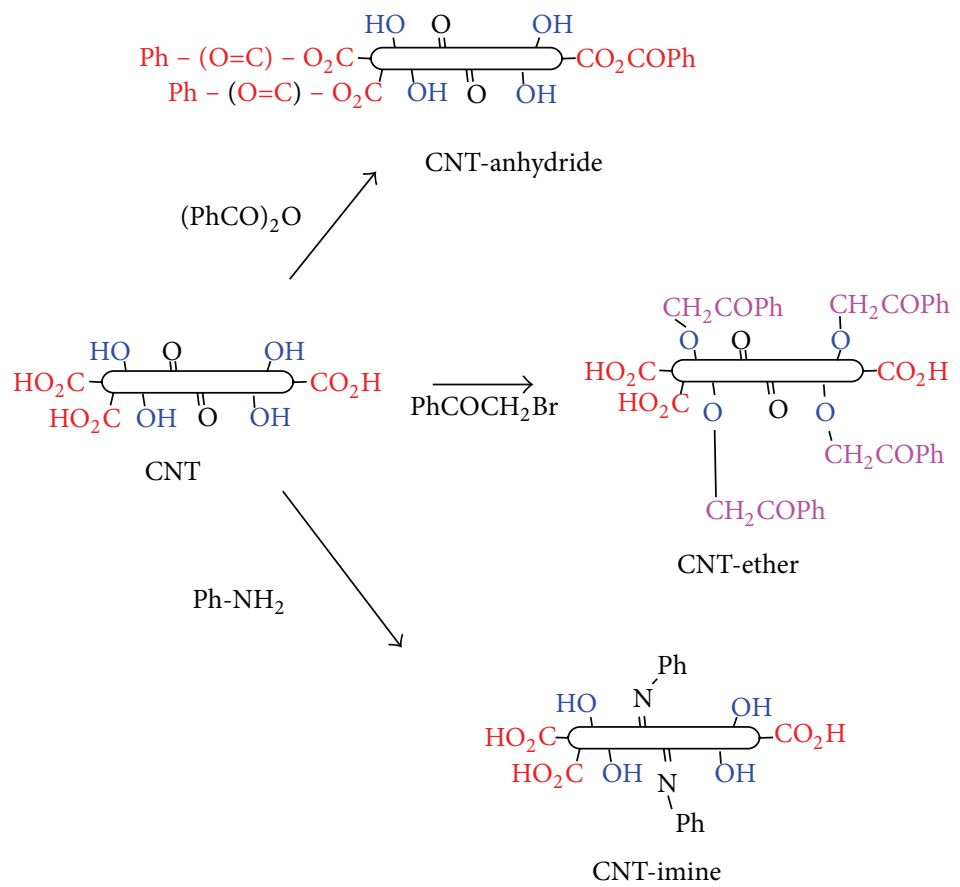

SCHEME 5: Derivatisation of CNTs to mask selectively oxygenated functional groups to assess the nature of the active sites. It was found that CNT, CNT-anhydride, and CNT-ether perform with similar catalytic activity four times higher than that of CNT-imine.

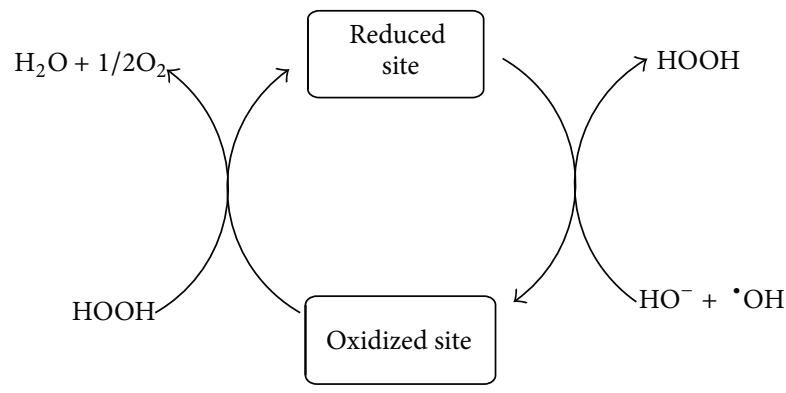

Scheme 6: Catalytic hydrogen peroxide decomposition (dismutation) mediated by a redox site that could be present in a carbon nanoform.<smiles>C1CCCCC1</smiles>

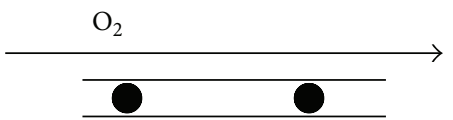

(Fe NP)@CNT

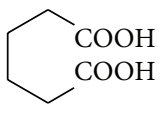

Adipic acid
SCHEME 7: Aerobic oxidation of cyclohexane to adipic acid catalysed by Fe NPs incorporated inside CNTs ((Fe NP)@CNT).

subjected to intensive study has been storage of hydrogen into a chemical compound that can release hydrogen on demand at moderate temperatures with the assistance of a catalyst. After being used, the residual product resulting from hydrogen release should also regain catalytically hydrogen forming the initial hydride. One of the preferred metal hydrides for this process has been $\mathrm{LiBH}_{4}$ (1). It has been found that CNTs can release up to $8.8 \mathrm{wt} \%$ of hydrogen from $\mathrm{LiBH}_{4}$ under mild conditions [61]. However, comparison with $\mathrm{GO}$ and $\mathrm{r}-\mathrm{GO}$ indicates that the hydrogen release using G-based materials is about $1 \%$ higher than that of using CNTs. This comparison suggests that defects and residual oxygen functionalities are acting as catalytic centers in this process and that CNTs could have a lower density of this type of sites:

$$
\mathrm{LiBH}_{4} \rightleftharpoons \mathrm{LiH}+\mathrm{B}+1.5 \mathrm{H}_{2}
$$

One of the possibilities that CNTs offer in catalysis is their use as supports of metal NPs. Pd NPs supported on MWCNTs have been employed as catalyst for hydrogenation, oxidation, and $\mathrm{C}-\mathrm{C}$ coupling reactions [62-66]. The activity of $\mathrm{Pd} / \mathrm{MWCNTs}$ has been compared to that of palladium supported on $\mathrm{ACs}(\mathrm{Pd} / \mathrm{C})$, and it was found that the turnover number with respect to $\mathrm{Pd}$ was higher for $\mathrm{Pd} / \mathrm{MWCNTs}$ than that for $\mathrm{Pd} / \mathrm{C}$ for some of these reactions [33]. It was considered that the interaction between the graphene wall of the support with $\mathrm{Pd}$, together with the morphology of the nanotubes, is beneficial to increase the catalytic activity of Pd NPs for those reactions in which the Pd particle size is a key parameter controlling the catalytic activity. In contrast, Pd supported on MWCNTs were much less active than Pd supported on charcoal for those reactions such as hydrogenation of cinnamaldehyde and oxidation of benzyl alcohol that are less sensitive to the average particle size of Pd (Scheme 8).

The strong metal-support interaction arising from overlapping of the extended $\pi$ system of the graphene wall of CNTs and the orbitals of metal clusters has also been claimed as being responsible for the formation and stabilization 
Reactions catalyzed by palladium supported on MWCNTs

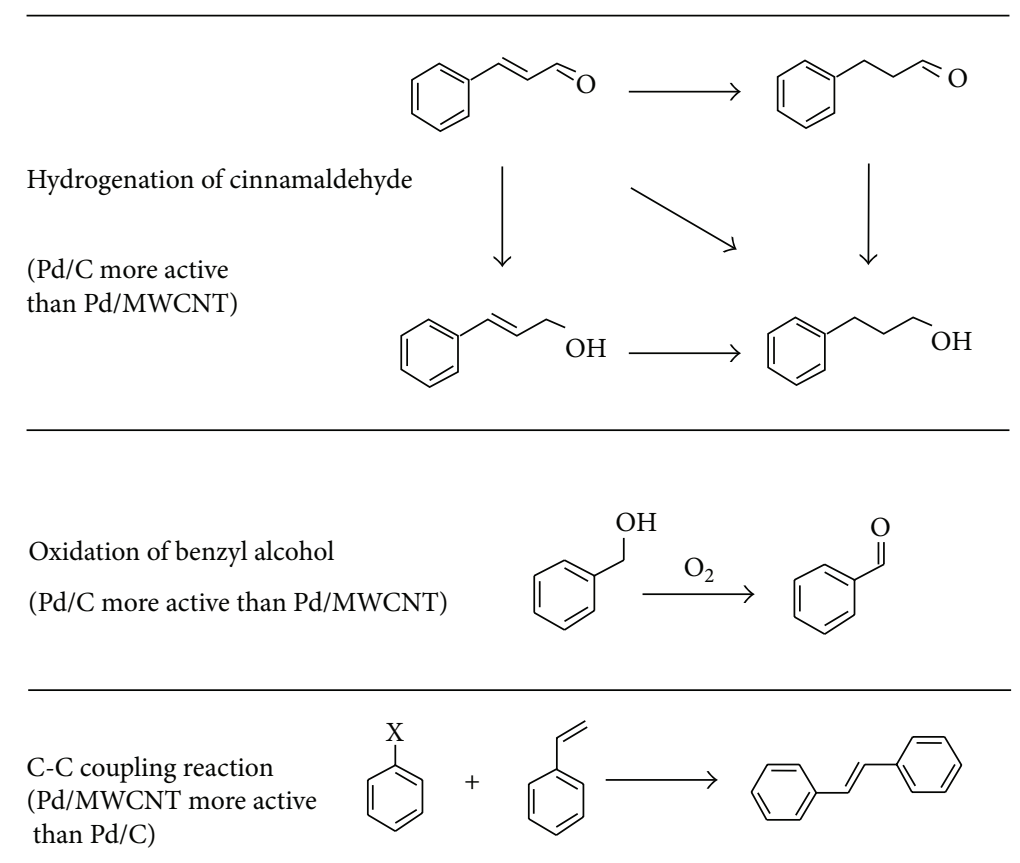

SCHeme 8: Comparison of the catalytic activity of Pd NPs supported on MWCNTs or on ACs (based on [33]).

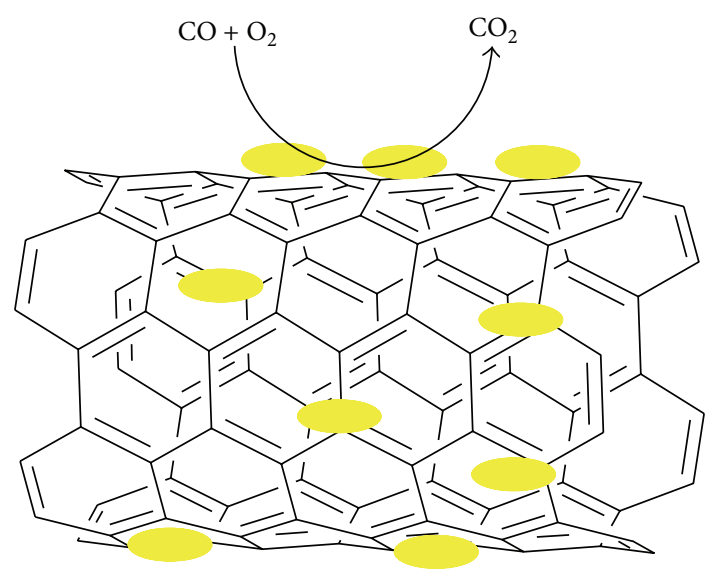

SCHEME 9: Pictorial representation of Au NPs supported on modified SWCNT acting as catalyst for the aerobic oxidation of CO.

of small Au clusters on SWCNTs and for the remarkable catalytic activity towards molecular oxygen dissociation (Scheme 9) [67, 68]. Supported Au NPs are highly active and selective catalysts for the aerobic oxidations of various functional groups [69], and the experimental data indicate that the support always plays an important role in the catalytic activity of Au NPs and in the reaction mechanism. In the present case, Au NPs supported on SWCNTs are highly active for the low temperature $\mathrm{CO}$ oxidation and theoretical calculations at the DFT level indicate that this remarkable catalytic activity should be mainly due to the ability of Au
NPs on SWCNTs for molecular oxygen dissociation resulting in the generation of Au oxide clusters highly dispersed on the material. Recently, Corma et al. have shown that it is possible to prepare and characterize clusters of a few $\mathrm{Au}$ atoms on the surface of modified MWCNTs and that these clusters between 5 and $10 \mathrm{Au}$ atoms are exceedingly active for the aerobic oxidation of thiophenol to diphenyldisulfide [70]. It is clear that this type of interaction $\pi$-d between CNTs and metal NPs is currently underestimated and other remarkable examples observing an increase in the catalytic activity can similarly be achieved in other cases. The curvature of the graphene walls and the presence of defects (oxygen functional groups and carbon vacancies) or heteroatoms should constitute powerful tools to tune the electron density on the metal NP.

Besides the use as support of metal NPs, CNTs can also be employed as platforms to anchor metal complexes that can act as catalytic sites. CNTs conveniently cut and purified can form permanent inks in aqueous solutions or organic media, but once used as catalysts they can be recovered by filtration. In this way, the active sites will be highly dispersed in the reaction media during the reaction but can be recovered at the end of the process and the catalyst recycled ("pseudohomogeneous catalyst"). An example of this strategy has been the anchoring of a vanadyl salen complex that has been used as catalyst for the cyanosilylation of aldehydes (Scheme 10) $[71,72]$.

An important point in this approach is characterization of the integrity of the metal complex and this is better guaranteed if anchoring of the metal complex to SWCNTs is carried out in the last step of the preparation of the material 

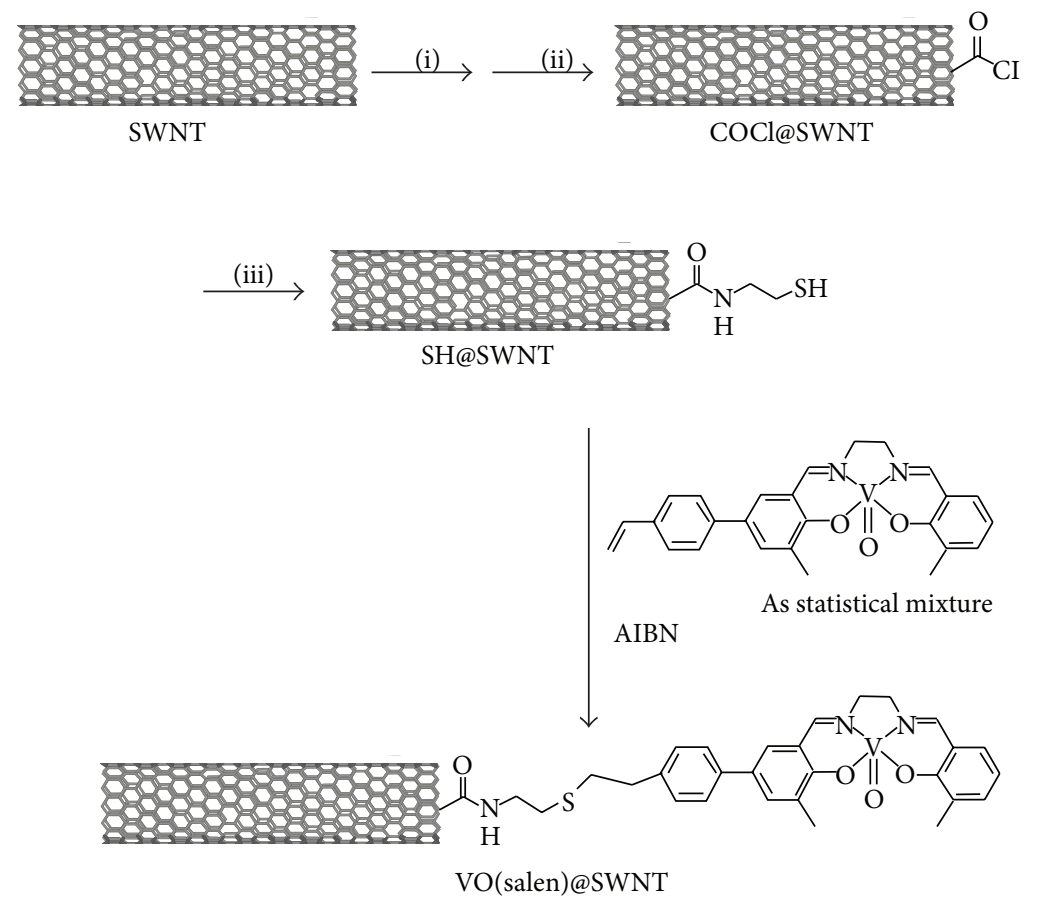

Scheme 10: Synthesis of a vanadyl salen complex anchored to SWCNTs. Reagents and conditions: (i) $3 \mathrm{M} \mathrm{HNO}_{3}$, reflux, $24 \mathrm{~h}$; (ii) SOCl , DMF, $60^{\circ} \mathrm{C}, 24 \mathrm{~h}$; and (iii) 2-aminoethanethiol hydrochloride, $\mathrm{Et}_{3} \mathrm{~N}, \mathrm{CH}_{2} \mathrm{Cl}_{2}, 45^{\circ} \mathrm{C}, 48 \mathrm{~h}$.

since all the previous intermediates can be purified and fully characterized by routine analytical and spectroscopic tools commonly employed in organic chemistry. Compared to AC, the use of short SWCNTs has the advantage of a well-defined morphology and chemistry for covalent functionalization that can be based on the reactivity of carboxylic groups present predominantly at the tips and wall defects of the CNTs or on the reactivity of the graphene wall through specific cycloadditions such as the so-called Prato reaction or radical addition (Scheme 11) [73]. In the case of the vanadyl salen SWCNTs it was found that the system is reusable and the chiral version can induce the preferential formation of one enantiomer of the $\alpha$-cyano trimethylsilyl ether with high enantiomeric excess [71]. This area, however, still needs to be developed and further work is necessary to fully exploit the possibilities that CNTs offer as scaffolds to anchor covalently metal complexes including high dispersability, easiness of recovery, the interaction of substrates and sites with the graphene walls, either conducting or semiconducting, and the special morphology with long aspect ratio and high curvature of the graphene wall.

\section{G-Based Materials in Catalysis}

Compared to CNTs that are obtained by pyrolysis of adequate volatile carbon precursors on transition metal-containing catalysts (Fe and Co alloys or other possible metals) or by arc-discharge on graphite electrodes prepared adequately in such a way that they already contain the metal catalyst [36, $74,75]$, Gs can be prepared by many other ways, some of them are chemical methods [76]. Chemical procedures can be preferable because they generally allow the preparation of large quantities. Thus, one of the most popular ways to prepare G-based materials starts with graphite that is deeply oxidized using $\mathrm{KMnO}_{4}$ and $\mathrm{H}_{2} \mathrm{O}_{2}$ under strong acid conditions $\left(\mathrm{H}_{2} \mathrm{SO}_{4}, \mathrm{HNO}_{3}\right)$, followed by exfoliation and dispersion in an adequate solvent leading to GO suspensions [77]. GO has a tendency to undergo chemical reduction leading to a decrease in its oxygen percentage, typically about $50 \mathrm{wt} \%$ oxygen content for GO obtained from graphite oxidation, forming suspended materials with residual oxygen content that are generally denoted as reduced graphene oxide (rGO) [76].

Recently, we have reported a greener alternative to obtain $\mathrm{G}$ and doped Gs consisting in the pyrolysis in the absence of oxygen of biomass precursors such as modified alginates or chitosan (Scheme 12) $[35,78,79]$. Chitosan acts as single source of carbon and nitrogen and depending on the pyrolysis temperature $\mathrm{N}$-doped $\mathrm{G}$ can be obtained with various percentages of nitrogen, up to $8 \mathrm{wt} \%$, that decreases as the pyrolysis temperature increases. Also, alginate modified by boric acid leads upon heating at temperatures higher than $600^{\circ} \mathrm{C}$ in the absence of oxygen to B-doped G; the percentage of boron depends on the amount of borate in the precursor and on the pyrolysis temperature (Scheme 12) [35].

Pyrolysis of natural biopolymers tends to form graphitic carbon residues with loose stacking of the graphene sheets as evidenced by XRD. These graphitic carbon residues can be subsequently easily exfoliated without the need of oxidation [80]. Thus, no liquid chemical wastes are generated in the formation of doped $\mathrm{G}$ by biomass pyrolysis and, in addition, 


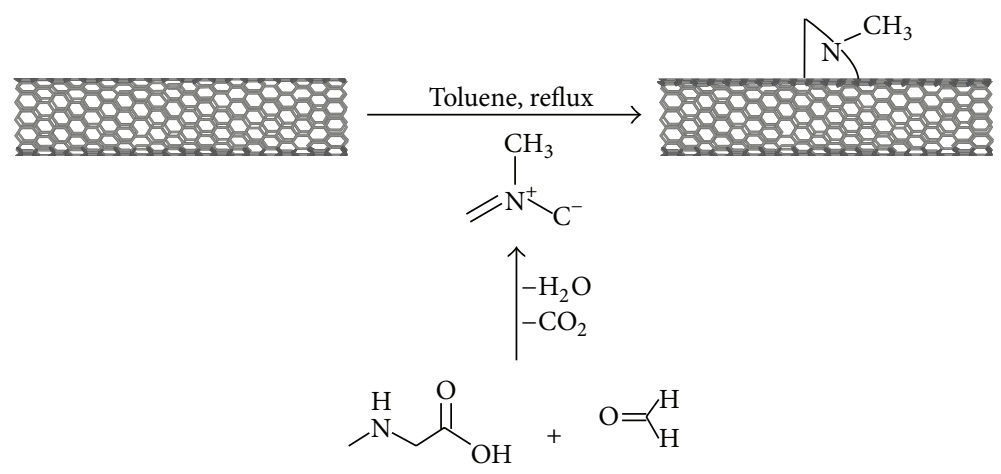

Scheme 11: Covalent functionalization of CNTs by dipolar cycloaddition ("Prato reaction") to the graphene walls forming a pyrrolidine linkage.

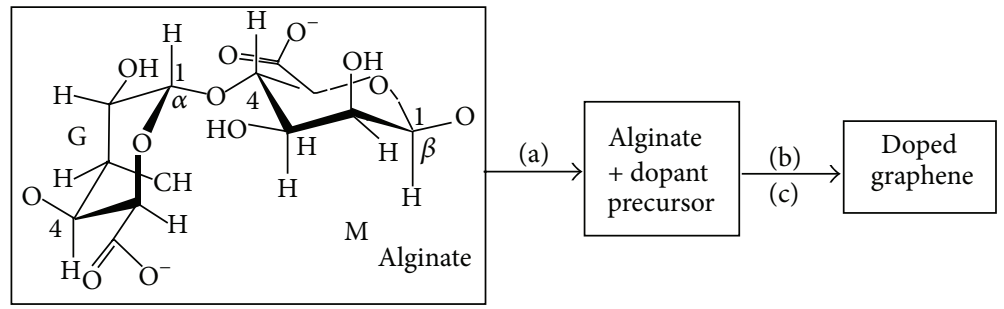

Scheme 12: General route for the synthesis of doped G by using alginate as G precursor that is modified by addition of a compound of the dopant element (a), followed by pyrolysis of the modified biopolymer in the absence of oxygen (b) and sonication in the presence of a liquid phase (c). The letters $\mathrm{G}$ and $\mathrm{M}$ correspond to the guluronic and maluronic monosaccharides of alginate.

only a natural biopolymer (typically considered as a valueless biomass waste) in combination or not of other dopant precursors is employed in the synthesis. In summary, either starting from graphite and submitting it to deep oxidation or starting from other precursors, $G$ materials are more easily available than CNTs and can be prepared in larger scale basically because they do not require catalysts to nucleate the dehydrogenative carbonisation of the walls.

One advantage of G-based materials is their large diversity and the opportunities to modify the $\mathrm{G}$ sheet by oxidation and doping with heteroatoms. In this sense, the group of Bielawski has pioneered in showing that GO can be a carbocatalyst for oxidation reactions (Scheme 13) [21].

Benzyl alcohols can undergo aerobic oxidation promoted by GO in the absence of metal [81]. Also, GO as acid carbocatalyst promotes dimerization and oligomerization of styrene [82, 83]. However, it has to be mentioned that impurities present in GO have to be surveyed as possible active sites responsible for the catalytic activity. Since GO preparation employs a large excess of $\mathrm{KMnO}_{4}$ and $\mathrm{H}_{2} \mathrm{SO}_{4}$, it could be possible that these chemicals (or some impurities accompanying them) may not have been removed completely from GO and that these impurities at the ppm level or above could be responsible for the catalysis in these reactions. For instance, our group has shown that GO can catalyze the room-temperature acetalization of aldehydes by methanol and the epoxide ring aperture (Scheme 14) and that this activity is related to the presence of sulphate groups anchored to $G[84,85]$. In accordance with the presence of impurities on GO and their role in catalysis, it has been found that exhaustive GO washings to the point in which the sulfur content becomes below ppms reduces significantly the catalytic activity of GO for these two processes [84, 85]. Based on this, it has been proposed that $-\mathrm{OSO}_{2} \mathrm{OH}$ groups anchored on GO sheets should be the active sites for these two acid-catalyzed reactions. The excellent activity of GO is a consequence of the high surface area, easy accessibility, and excellent dispersability of GO sheets. Comparison of the catalytic activity of GO obtained from Hummers oxidation with that of acetic acid reveals that HOAc is much less efficient to promote these two reactions that probably require sites of strong acidity. However, $-\mathrm{OSO}_{2} \mathrm{OH}$ groups are not permanently bonded to the $\mathrm{GO}$ sheets and can undergo hydrolysis. Therefore, upon reuse, a gradual decrease in the catalytic activity is observed [84]. In this sense, the need of complete analytical data of G-based materials should be emphasized since their catalytic activity can arise from $\mathrm{Mn}$, $\mathrm{Fe}$, or other metal impurities or adventitious acid sites well dispersed on the large surface area characteristic on singlelayer GOs.

More recently, our group has found that N-doped G or (B, $\mathrm{N}-$ ) codoped $\mathrm{G}$ are suitable carbocatalysts to promote aerobic oxidations [35]. Comparison of these doped G materials with the catalytic activity of undoped $G$ prepared following the same procedure suggests that this catalytic activity is due to the presence of the dopant elements. In comparison 


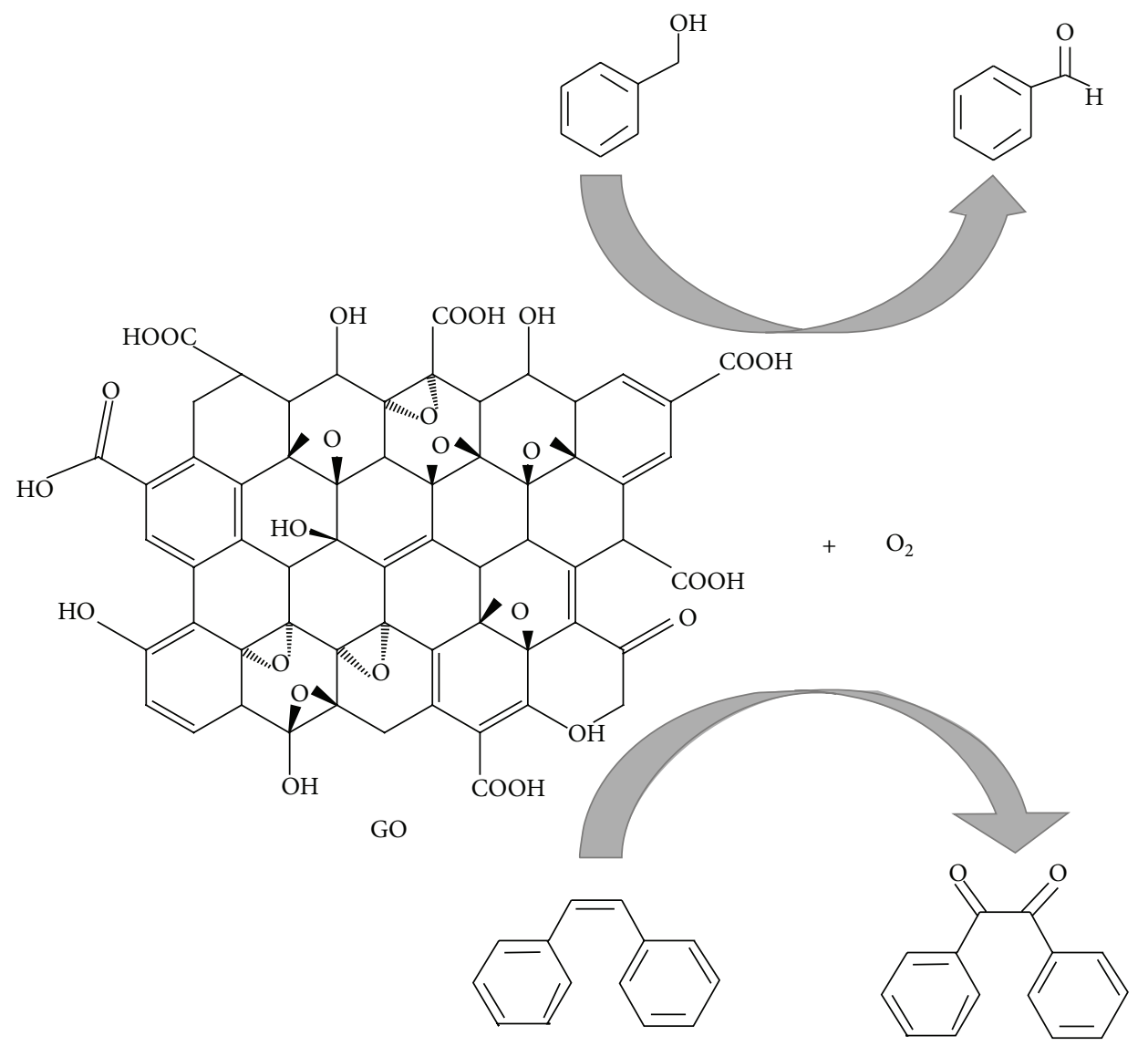

Scheme 13: Catalytic activity of GO to promote the aerobic oxidation of benzylic alcohols and cis-stilbene.

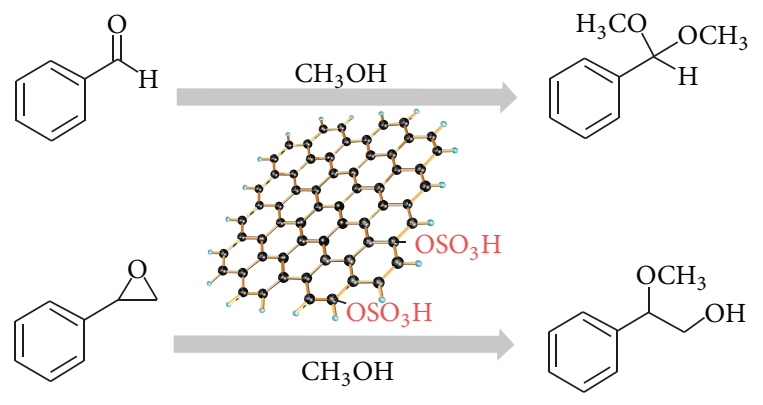

Scheme 14: Catalytic activity of rGO for the room temperature formation of dimethyl acetal and epoxide ring aperture due to the presence of residual sulfate groups anchored to the $\mathrm{G}$ sheet.

with $\mathrm{N}$-doping, doping with $\mathrm{B}$ atoms leads to a material with lower activity [35]. IR monitoring of the interaction of molecular oxygen with (N)G shows the appearance of a new band that has been attributed to some peroxyl groups on $\mathrm{G}$ [35]. Formation of this peroxyl group is reversible and mild heating and evacuation under reduced pressure lead to the disappearance of this band [35]. Other studies have also shown the ability of $\mathrm{N}$ atoms on $\mathrm{G}$ to activate molecular oxygen [86], and how this interaction can serve to promote aerobic oxidations of benzylic alcohols and hydrocarbons, although they may require the use of tert-butylhydroperoxide as initiator [35]. Overall, the above data shows the potential that the incorporation of dopants on the G sheet can have to produce active sites on the carbocatalysts as I have already pointed out for the case of CNTs (Scheme 3) [35].

Besides benzylic alcohols and hydrocarbons, styrene can also undergo aerobic oxidation by doped $G$ leading to oxidative $\mathrm{C}=\mathrm{C}$ bond degradation forming benzaldehyde or $\mathrm{C}=\mathrm{C}$ bond epoxidation accompanied by rearrangement of the epoxide to 2-phenylacetaldehyde (Scheme 15) [35]. The important observation here is that the product selectivity changes along styrene conversion. Thus, benzaldehyde is formed initially with almost complete selectivity, while styrene oxide appears at higher conversions but can reach selectivities over $60 \%$ at final reaction times [35].

These changes in product selectivity as well as the formation of benzaldehyde without induction period have led to proposing a mechanism for styrene oxide formation that is similar to the one assumed for oxidation with molecular oxygen using a transition metal complex or salt and aldehydes as cocatalysts [87]. According to this mechanism, when the concentration of benzaldehyde is sufficiently high, reaction of oxygen with benzaldehyde promoted by doped $G$ in the absence of metals will lead to the formation of benzoyl 


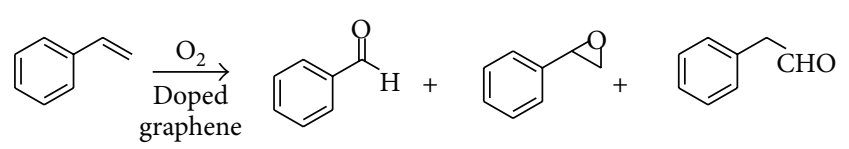

Scheme 15: Product distribution in the aerobic oxidation of styrene promoted by doped $\mathrm{G}$.

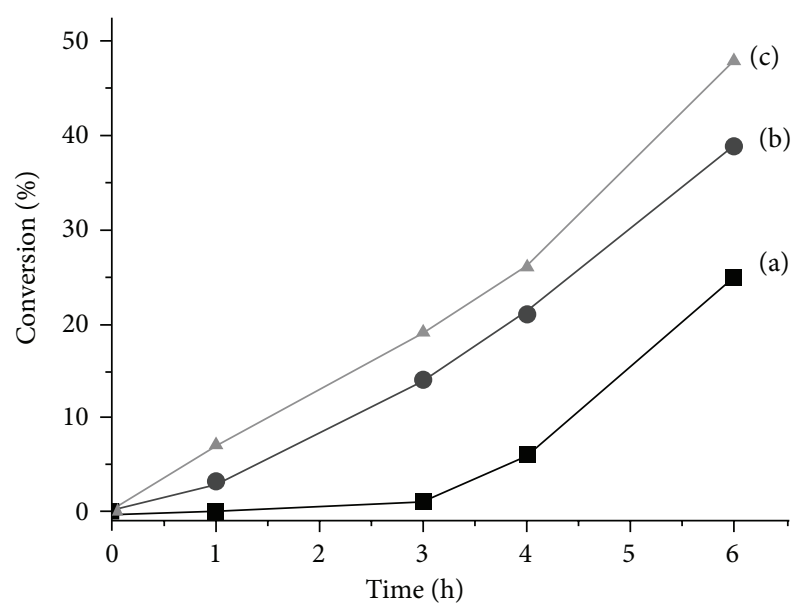

FIGURE 1: Time-conversion plots for the aerobic oxidation of styrene using $(\mathrm{N}) \mathrm{G}$ as catalyst in the absence (a) and in the presence of $2.5(\mathrm{~b})$ and $5 \mathrm{wt} \%$ (c) of benzaldehyde. Reaction conditions: styrene $(1 \mathrm{~mL})$, (N)G (10 mg), and oxygen purging through a balloon, $100^{\circ} \mathrm{C}$. Plot taken with permission from [35].

peroxides and peracids that will be the real oxidizing species leading to $\mathrm{C}=\mathrm{C}$ epoxidation. Experiments in which various amounts of benzaldehyde were added since the beginning of the reaction show that, under these conditions, styrene oxide is formed without any induction period (Figure 1).

As commented in the section of CNTs, also G-based catalysts exhibit activity for the decomposition of peroxide monosulfate and other peroxides $[52,88]$. The main application of these reactions has been decolorization of dyes present in aqueous solution. Peroxide monosulfate as reagent has the advantage over hydrogen peroxide in that the process can take place at neutral $\mathrm{pH}$ values and that the resulting sulfates radicals are highly reactive species attacking most of the organic compounds that could be present in water.

Besides oxidations, $G$ can also be used for reduction. Although obviously this reaction type has been much more frequently performed with catalysts containing noble metals, $\mathrm{G}$ in the absence of any metal can have also some activity. One of the favorite reactions for which the catalytic activity of $\mathrm{G}$ has been tested is the reduction of nitrobenzene and derivatives with $\mathrm{NaBH}_{4}$ [89-91]. In most of the cases, a large excess of $\mathrm{NaBH}_{4}$ (over 300 equivalents) was used. Although this large excess of $\mathrm{NaBH}_{4}$ is unrealistic for any application due to the relatively high price of this commodity chemical, it can be used as a benchmark reaction to rank the activity of the $G$ catalysts by using reaction conditions in which the kinetics becomes apparently of first order. In this way the value of the rate constant can quantitatively assess the activity of the catalyst. Another advantage of the reduction

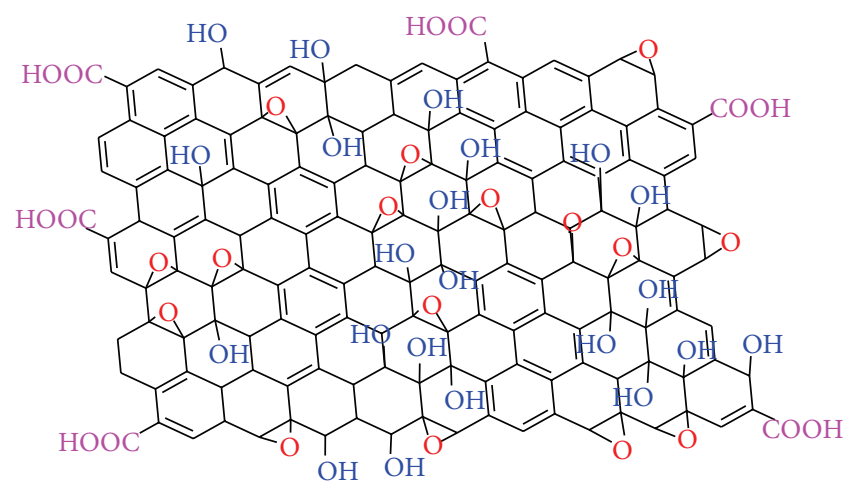

FIGURE 2: Model for GO showing the possible oxygenated functional groups and their location on the sheet.

of nitrobenzene to aniline as a model reaction is that using nitrophenol as probe under basic $\mathrm{pH}$ values, the reaction can be carried out in aqueous solution, highly compatible with GO and r-GO, and the course of the reaction can be simply monitored by following in UV/visible spectroscopy the decay and growth of the specific bands corresponding to nitrophenol and hydroxyaniline, respectively.

As commented previously in the case of CNTs, oxidative dehydrogenation of alkanes is a reaction that can be carried out also using G-based materials as catalyst [92]. In particular, $\mathrm{GO}$ has been reported as catalyst for the process. It should be commented that there are different models of GO that try to fit with spectroscopic and analytical data for this material. These models indicates the type of oxygenated functional groups that should be present in highly oxidized GO (Figure 2). The functional groups include epoxide, ether, hydroxyl, and carboxylic acid functionalities and basically have to explain the high oxygen content of GO that can be even above $50 \%$ in weight as I have already pointed out. This high oxygen content present in GO determines that the active sites that have been proposed for the oxidative dehydrogenation of propane on CNTs (quinone-like moieties) could not be the same as those responsible for the same reaction in GO.

In fact it has been proposed that in the case of GO, epoxy groups should be mainly responsible for the process [92]. In a certain way, GO would act in the reaction mechanism for the oxidative dehydrogenation analogously to the wellestablished Mars van Krevelen mechanism occurring in nonstoichiometric metal oxides. In these nonstoichiometric oxides oxygen from the solid lattice is reversibly transferred to the substrate causing its oxidation and, then, is replenished by the oxidizing reagent [93]. According to this analogy, oxygen atoms of the epoxide groups present on GO will form water by reaction with the propane, but, in a subsequent step, epoxides will be formed again by reaction with molecular oxygen.

One interesting application of G-based materials is to act as catalyst in the combustion of nitromethane and other high energy fuels for rocketry, thus increasing the power that the fuel can deliver to the engine. Combination of theoretical and experimental data indicates that defects on the $G$ sheet and dangling bonds are responsible for the generation of 
nitromethyl radicals that subsequently react with adsorbed oxygen and also for the decomposition of peroxide intermediates $[94,95]$. It could be interesting also to determine if this catalytic activity of $\mathrm{G}$ in combustion reactions can be applied to conventional fuels, such as gasoline or diesel, where the combustion of $\mathrm{G}$ could boost the octane or cetane number of fuels.

Although the use of $G$ materials as carbocatalysts is developing currently at a very fast pace, it is clear that at the present, the most widely use of $G$ in catalysis is as support of metal NPs. In this type of reactions, G can cooperate to the process at least in four different ways. The first one is providing a material with a very large surface area allowing a good dispersion of the metal NPs (estimated about $2630 \mathrm{~m}^{2} \times \mathrm{g}^{-1}$ for fully exfoliated, single-layer material) [96]. In addition, a second possible effect is the strong metal-G interaction that takes place particularly at defects and in the position in which heteroatoms are located in doped Gs [97, 98]. The extended $\pi$ orbital of $G$, especially in certain areas, is particularly suitable for overlapping with the $d$ orbitals of transition metals leading to charge transfer phenomena between the metal and the support. This orbital overlap also determines a high affinity of $\mathrm{G}$ for metals, minimizing leaching of the metal from the surface to the liquid phase and also reducing particle growth and agglomeration. In this case, the key point is to show how the presumably strong $\pi$-d interaction between the $\mathrm{G}$ sheet and the metal atoms modifies the intrinsic catalytic activity of the metal NPs with respect to other supports.

A third general effect that has been frequently claimed to rationalize the excellent performance of the catalytic activity of metal NPs supported on G has been the strong adsorption capacity of $\mathrm{G}$ for substrates and reagents, bringing them in close proximity to the active sites and even also transferring electrons to them.

A fourth way in which $\mathrm{G}$ can contribute to the catalysis in which metal NPs are the main active sites is by providing acid, base, or other types of sites that can cooperate in certain steps of the reaction mechanism. The frequently observed consequence of the use of $G$ as support of metal NPs is a very good dispersability of the material in the reaction medium that derives from the single-layer morphology and subnanometric dimensions of the $\mathrm{G}$.

Comparison of the activity and selectivity of G-supported metal NPs with that exhibited by other related materials and, particularly, metal supported on ACs is necessary in order to fully delineate the advantages of using $\mathrm{G}$ sheets as supports. The presence of active sites on the $G$ sheet combined with the catalysis by the metal could lead to the development of bifunctional catalysts with activity in tandem reactions in which two or more processes occur in a single step.

The flat surface of $G$ sheets is particularly suitable for the interaction with metal NPs and Pd, $\mathrm{Au}, \mathrm{Pt}$, and Ru have been among the preferred examples for their use in catalysis [99]. At the moment, although there is a large number of examples for preparation of supported metal NPs on G, their application in catalysis is still relatively limited. It is expected that the numbers of examples will grow in the near future, applying Gs not only as catalysts oxidation, reductions, and couplings, but also for novel reactions in the field of reversible hydrogen release/uptake. In the case of Au NPs supported on Gs there are some examples showing their activity as reduction catalysts for the transformation of aromatic nitro groups into amines using sodium borohydride as reagent [100]. Similarly, Pt NPs have been supported on $\mathrm{G}$ and used as oxidation and hydrogenation catalysts that are reaction types of general importance in industry and organic chemistry [101]. Pd NPs supported on Gs have been the preferred pseudohomogeneous catalyst for coupling reactions [102].

Theoretical studies suggest that defects on G should favour the interaction with supported Pt NPs [103]. Computational $a b$ initio calculations have led to proposing that $\mathrm{Pt}$ supported on defect-engineered G should be more tolerant compared to free Pt NPs to the poisoning by $\mathrm{CO}$, since it should show a higher affinity for $\mathrm{H}_{2}$ [104]. This lower tendency to $\mathrm{CO}$ poisoning is of importance for the development of fuel cells and must be corroborated by experimental measurements [105].

Pt NPs supported on rGO can be obtained by solvolysis using ethylene glycol as reductant and stabilising agent [106-108]. The average particle size of Pt NPs prepared in ethylene glycol can be around $3 \mathrm{~nm}$ and they can exhibit oriented 1.1.1 facets. This material performs for hydrogenation of nitrobenzene to aniline over 12 times more efficiently than an analogous Pt catalyst using MWCNTs as support. Furthermore, the catalytic activity at $0^{\circ} \mathrm{C}$ of Pt-rGO is about 20 times higher than the activity of Pt supported on AC. This enhanced catalytic activity of Pt-rGO is proposed to arise from the high dispersion of Pt clusters on rGO and from the dispersability of this material in the reaction mixture [101].

Electrical conductivity is one of the main properties of $\mathrm{sp}^{2}$-forms of carbon allotropes and particularly of G-based materials. This electrical conductivity can serve to develop electrocatalysts $[86,109]$. Pt NPs supported on G sheets of small dimensions (G quantum dots (GQDs)) have also been prepared by solvolysis with ethylene glycol of $\mathrm{PtCl}_{4}{ }^{-}$on nanosized GQDs obtained by acid etching of carbon fibers [110]. The resulting material exhibits high activity as electrode for the electrochemical oxygen reduction, where the target is to reduce as much as possible the overpotential needed for this electrochemical process [111]. It was found that Pt-GQD shows an onset potential for oxygen reduction of $+1.05 \mathrm{~V}$, that is, $70 \mathrm{mV}$ more positive than the onset potential observed for an analogous electrode prepared with Pt supported on AC [111]. In fact, due to the electrical conductivity, G materials containing or not metal NPs have been widely used as electrocatalysts, but this area has been covered extensively in recent reviews and the reader is addressed to them for a complete coverage [112-115].

\section{Diamond Nanoparticles (DNPs) as Support}

DNPs are affordable and commercially available (Aldrich, CAS: 7782-40-3). DNPs can be prepared by milling of diamond powders or by explosive detonation [28]. In the last 
case, the commercial samples have DNPs embedded in a matrix of amorphous carbon ("soot") and it is necessary to treat the samples to etch this amorphous soot matter. DNPs from milling have generally much larger particle size than samples obtained by detonation that are smaller than $10 \mathrm{~nm}$. Considering the importance of having small particle sizes, DNPs from detonation should be preferred as support in catalysis provided that they are liberated from the soot.

In the previous shown cases of CNTs and G allotropic forms, the carbon atoms have $\mathrm{sp}^{2}$ atomic orbitals and a strong interaction due to the overlap of extended $\pi$ orbitals of CNTs or G materials with substrates or metal NPs should play a key role in the catalytic activity. In contrast, in the case of DNPs the carbons are mainly $\mathrm{sp}^{3}$ with surface $\mathrm{OH}$ groups and no $\pi-\pi$ or $\pi$-d overlapping can take place. Moreover, a large percentage of the surface of DNPs can be highly inert and can be envisioned better as devoid of interactions with the active sites or metal NP. This robustness and inertness of DNPs can be, however, beneficial for their use as support to promote some reactions in which highly aggressive species that can react with the support are going to be formed. Thus, the current state of the art does not consider DNPs as carbocatalysts, since there is no a clear view of which type of sites could be present in $\mathrm{sp}^{3}$ carbons, but, on the other hand, they complement CNTs and Gs as support, since they provide and inert and robust surface that, however, can immobilize metal NPs by the presence of occasional OH groups.

One example of the beneficial use of DNPs as supports of metal NPs is in the catalytic Fenton reaction for the degradation of the organic pollutants in water by hydrogen peroxide $[116,117]$. DNPs can be hydrophilic materials when the population of surface hydroxyl groups is large. It is in these surface $\mathrm{OH}$ nests where metal NPs are anchored. The density of these hydroxyl groups can be diminished to meet the optimal density required to interact with the metal NPs by reductive treatments with hydrogen at temperatures above $300^{\circ} \mathrm{C}$ that converts $\mathrm{C}-\mathrm{OH}$ into $\mathrm{C}-\mathrm{H}$ groups [118]. Turnover numbers as high as 500,000 have been determined for $\mathrm{Au}$ supported on DNPs in the degradation of phenol taken as model pollutant $[116,117,119]$. For this reaction at acid $\mathrm{pH}$ values, almost quasistoichiometric $5: 1$ equivalents of $\mathrm{H}_{2} \mathrm{O}_{2}$ to substrate are needed $[116,117,119]$. These conditions are remarkable, since very frequently reported Fenton catalysts use $\mathrm{H}_{2} \mathrm{O}_{2}$ excesses as large as 10,000 [116, 117, 119]. Apparently, the key point of the excellent catalytic activity of the Au-DNP as catalyst is the combination of the lack of spurious $\mathrm{H}_{2} \mathrm{O}_{2}$ decomposition characteristic of the catalytic behavior of $\mathrm{Au}$ NPs and the fact that ${ }^{\circ} \mathrm{OH}$ radicals formed in the process are free to diffuse into the solution, not remaining surface-bound as it happens with many other solid Fenton catalysts based on metal (typically Fe) supported on inorganic or organic solids (Scheme 16) [120, 121].

One of the undesirable limiting conditions of the Fenton chemistry that should be overcome is the need of acidic $\mathrm{pH}$ values, typically below 5 units, to occur [122]. For many applications, it will be important to effect the Fenton reaction at neutral $\mathrm{pH}$, since it is not possible to adjust the $\mathrm{pH}$ value for large water volumes or stream flows. Operation of Fenton

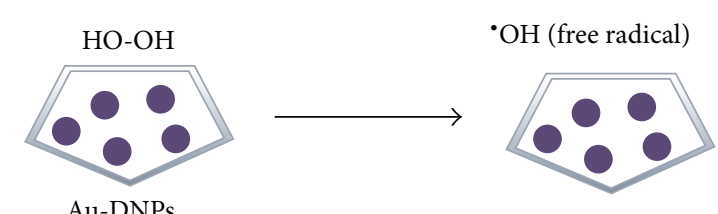

(a)

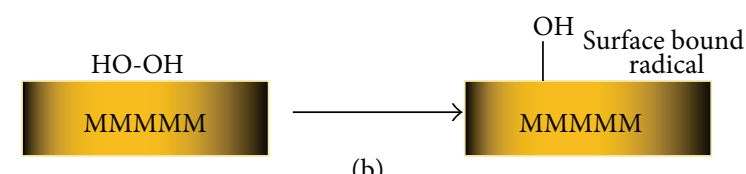

(b)

Scheme 16: Pictorial illustration of the characteristic catalytic activity of $\mathrm{Au}-\mathrm{DNP}$ generating free ${ }^{\circ} \mathrm{OH}$ radicals due to the inertness of its surface (a) in contrast to surface-bound ${ }^{\circ} \mathrm{OH}$ radicals (b).

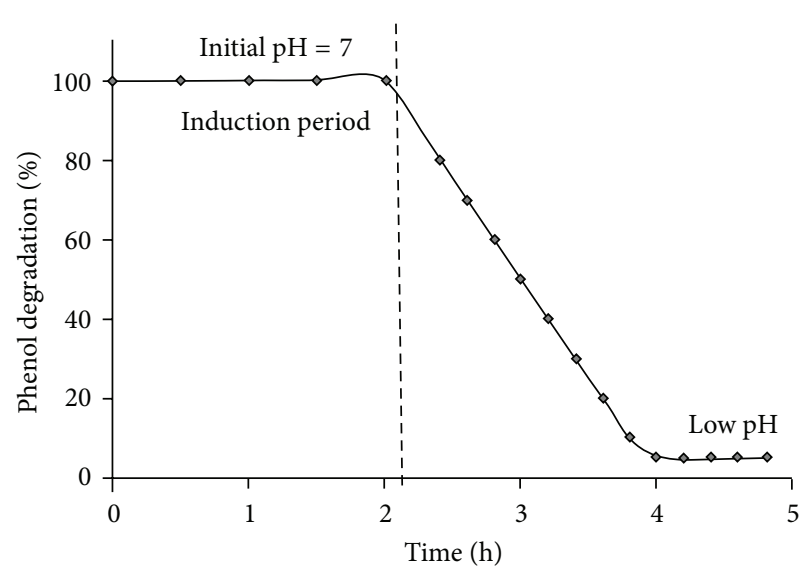

FIGURE 3: Representative example of an ideal time conversion plot for the phenol disappearance in the catalytic Fenton degradation by $\mathrm{H}_{2} \mathrm{O}_{2}$ using Au-DNP as catalyst in the dark in the absence of buffers. The reaction is initiated at neutral $\mathrm{pH}$, exhibiting an induction period. Once the reaction starts, there is a decrease in the $\mathrm{pH}$ value up to 3.5 due to the formation of polycarboxylic acids that accelerates the reaction.

catalysis at neutral $\mathrm{pH}$ can only be achieved using a very large excess of $\mathrm{H}_{2} \mathrm{O}_{2}$ and, if there are not buffers in the solution and for batch reactions, it is frequently observed that after an induction period characterized by a slow start up of the reaction an acceleration occurs (Figure 3 ). This often remarkable increase in the reaction rate is mainly due to the fact that the $\mathrm{pH}$ of the solution becomes spontaneously acidic as soon as some phenol decomposes due to the formation of carboxylic acids that are the degradation byproducts. It was, however, observed that in the case of Au-DNPs the reaction can take place at initial neutral $\mathrm{pH}$ values if the reaction is illuminated with solar light or artificial visible light $[116,117]$. The reason for this photoinduced process is that Au NPs exhibit a surface plasmon band at $\lambda_{\max } 560 \mathrm{~nm}$, and visible light absorption at this wavelength can promote electron injection from excited $\mathrm{Au}$ NPs to $\mathrm{H}_{2} \mathrm{O}_{2}$, leading to ${ }^{\circ} \mathrm{OH}$ radicals even in this unfavourably high $\mathrm{pH}$ range (Scheme 17) $[116,117]$. 


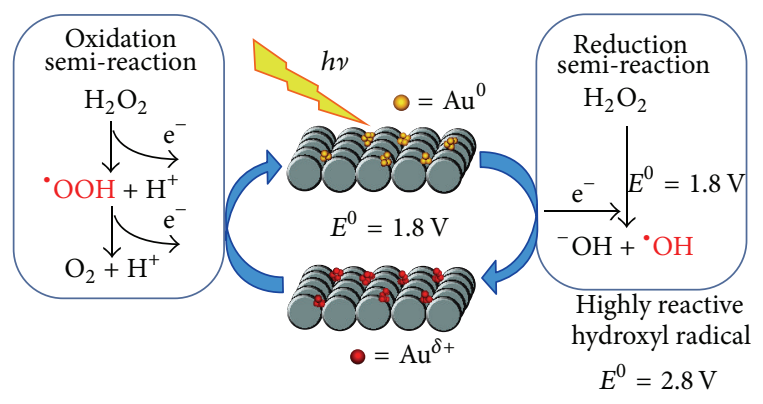

Scheme 17: Proposed mechanism for the photoinduced catalytic Fenton generation of ${ }^{\circ} \mathrm{OH}$ radicals at neutral $\mathrm{pH}$ values by visible light irradiation of Au-DNPs. The light is absorbed by Au NPs that exhibit a visible band at about $560 \mathrm{~nm}$ (surface plasmon band). Light absorption triggers electron ejection that causes the reduction of $\mathrm{H}_{2} \mathrm{O}_{2}$ and formation of ${ }^{\circ} \mathrm{OH}$ radical.

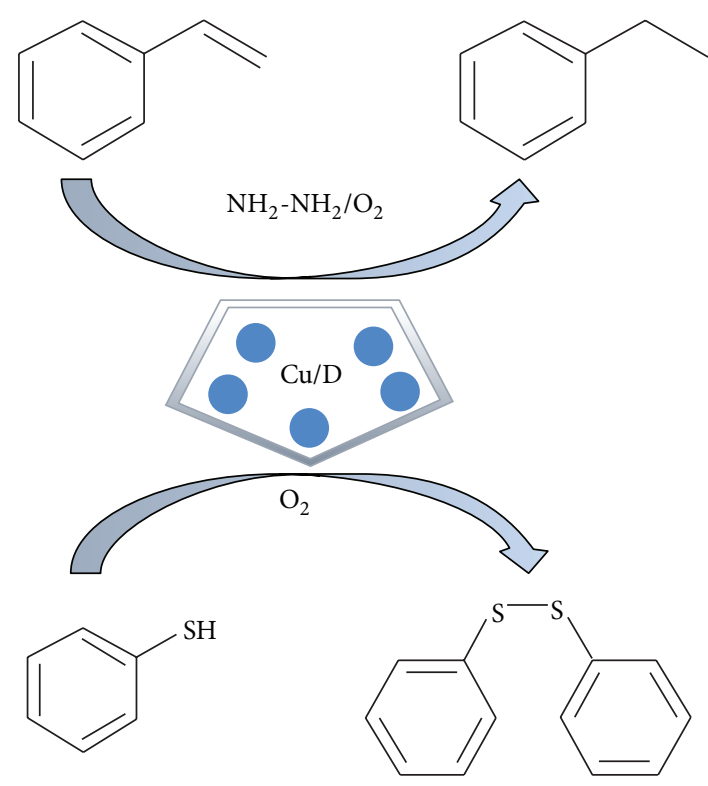

Scheme 18: Catalytic activity of $\mathrm{Cu}-\mathrm{DNP}$ for the $\mathrm{C}=\mathrm{C}$ double bond hydrogenation by hydrazine in the presence of oxygen and the aerobic oxidative coupling of thiophenol to diphenyldisulfide.

Alternatively or coincidentally, irradiation at the $\mathrm{Au}$ surface plasmon band can induce local heating near the $\mathrm{Au}$ NPs that initiate a thermally induced Fenton reaction [116, 117]. It has been reported based on estimation of the reaction rates and activation energies that irradiation can induce in the submillisecond time scale local temperatures as high as $300^{\circ} \mathrm{C}$ [123].

Recently the use of DNPs as supports of metal NPs has been extended by developing DNP-supported Cu NPs that are efficient catalysts for the aerobic oxidation of thiols to disulfides [124] and for the hydrogenation of $\mathrm{C}=\mathrm{C}$ double bonds by hydrazine (Scheme 18) [125]. As in the case of the Au-DNPs, the key feature to understand the excellent

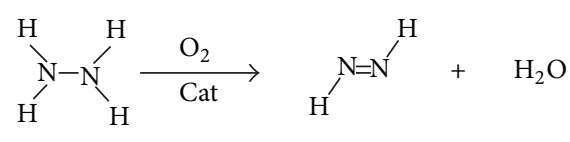

Hydrazine Diimide

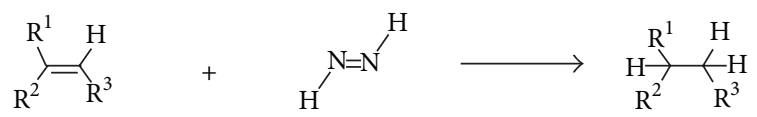

SCHeme 19: Proposed mechanism for the hydrogenation of $\mathrm{C}=\mathrm{C}$ double bonds by hydrazine under aerobic conditions promoted by $\mathrm{Cu}-\mathrm{DNP}$ as catalyst.

catalytic activity of $\mathrm{Cu}$ DNPs is the small particle size of the metal NP (in the subnanometric size) and the inertness of the surface. Thus, using hydrazine as reducing agent for the hydrogenation of styrene, $\mathrm{Cu}$-DNPs is far more active than other metal NPs including Pd and Pt or other supports such as ACs [126]. This higher activity of $\mathrm{Cu}$ NPs over precious metals is interesting from the point of view of reducing the dependency of catalysis on expensive noble metals. The reaction mechanism of Cu-DNP catalysed hydrazine reduction involves presumably the intermediacy of diimide generated by aerobic oxidation of hydrazine (Scheme 19). In fact, even though this reaction is a reduction, it requires the presence of oxygen to occur. Diimide (Scheme 19) is a highly reactive intermediate that spontaneously decomposes and can be envisioned as the precursor of $\mathrm{H}_{2}+\mathrm{N}_{2}$. The use of hydrazine combined with $\mathrm{Cu}$-DNPs as catalyst can be convenient for some applications avoiding manipulation of hydrogen gas.

$\mathrm{Cu}$-DNPs have also been found to be a recyclable catalyst for the selective oxidation of thiols to disulfides by molecular oxygen [124]. The interesting point here is that, on one hand, thiols are typical poisons of noble metals such as palladium and gold and, on the other hand, they tend to form different oxidation products including sulfenic and sulfonic acids. Thus, Cu-DNPs appear to be ideal catalyst that does not undergo deactivation and exhibits selectivity towards disulfide. TON values as high as 5,700 have been measured for the oxidation of thiophenol to diphenyl disulfide with the $\mathrm{Cu}$ DNP catalyst being reusable at least in four cycles at $\mathrm{PhSH} / \mathrm{Cu}$ mol ratio of 5772 with turnover frequency of $825 \mathrm{~h}^{-1}$ [124].

This behavior of $\mathrm{Cu}-\mathrm{DNPs}$ and its stability contrasts, for instance, with the performance of $\mathrm{Cu}$-containing metal organic frameworks such as $\mathrm{Cu}_{3}$ (BTC) 2 (BTC: 1,3,5-benzenetricarboxylate) that undergoes complete decomposition under similar conditions [127-129]. Metal organic frameworks are microporous crystalline solids that are used as catalysts for a wide range of organic [130] reactions including alcohol [131] and alkane aerobic oxidations [132]. However, metal organic frameworks and in particular $\mathrm{Cu}_{3}(\mathrm{BTC})_{2}$ may not be stable in the presence of thiols [133]. This comparison illustrates again the robustness of metal supported DNPs catalysts with regard to other alternative solids.

Besides being used as supports of noble metal and $\mathrm{Cu}$ NPs, oxidized DNPs have been also been used as supports of other first-row transition metal oxides that exhibit catalytic 


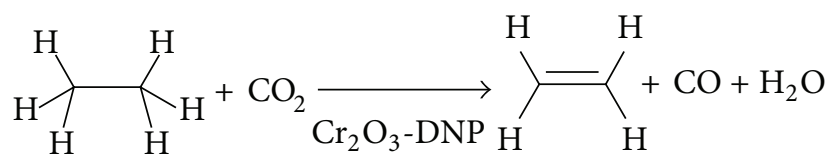

SCHeme 20: Oxidative dehydrogenation of ethane by $\mathrm{CO}_{2}$.

activity for hydrocarbon dehydrogenation or oxidation using $\mathrm{CO}_{2}$ as oxidizing reagent. I have shown previously that commercially available DNPs samples should preferably be oxidized to remove amorphous soot matter. This process generates a large density of oxygenated surface functional groups that can be undesirable to stabilize small metal NPs. For this reason, another alternative to remove this amorphous carbon contaminating DNPs could be initial hydrogenation of commercial diamond powder at high temperatures under pure hydrogen stream and, then, the process should be followed by oxidation with diluted molecular oxygen at $450^{\circ} \mathrm{C}$. This pretreatment is very important in order to control the properties of the external DNP surface that after the treatment contains carbonyl groups and ethers. It is, however, very likely that partial combustion of DNP surface could lead also to hydroxyl and carboxylic groups that can interact by sharing the oxygen with metal oxide clusters on the surface and, therefore, the conditions and time of the treatment can have a considerable impact on the performance of the resulting DNP as catalyst.

Using this type of DNP powders obtained by hydrogenation and oxidation as support, Nakagawa et al. have deposited metal NPs on the surface by wet impregnation of the corresponding metal salt followed by calcination at $450^{\circ} \mathrm{C}$ under air [134]. Depending on the nature of the metal oxide, the resulting DNP containing metal oxide NPs exhibits distinctive catalytic properties for various reactions of hydrocarbons with $\mathrm{CO}_{2}$.

For instance, Ni-DNP is able to promote dry reforming of methane (see (2)) making methane conversion reach about $25 \%$ at $600^{\circ} \mathrm{C}$ without deposition of elemental carbon on the catalyst [134]. It was proposed the catalytically active species in this dry reforming should be Ni NPs that must be formed from $\mathrm{NiO}$ at the initial stages of the reaction. The weak interaction of $\mathrm{NiO}$ with the surface of DNPs will be responsible for the easy generation of Ni NPs in the course of the reaction and therefore of the catalytic activity:

$$
\mathrm{CH}_{4}+\mathrm{CO}_{2} \underset{\mathrm{NiO}-\mathrm{DNPs}}{\longrightarrow} \mathrm{CO}+2 \mathrm{H}_{2}
$$

In another work, the partial oxidation of methane has been carried out using as catalyst Ni or Co NPs supported on DNPs. The catalysts were prepared by impregnation of DNP powders with the required amount of the metal salt followed by water evaporation and calcination at open air at $450^{\circ} \mathrm{C}$. The catalytic activity data show that Ni-DNP performs better than Co-DNP and significantly better than other analogous catalysts of these two metals on different supports, reaching conversions of $32 \%$ at temperatures of $700^{\circ} \mathrm{C}$ [135]. It was determined that at this temperature no carbon deposition on the catalyst occurs and, therefore, the activity of the catalyst remains steady without deactivation. Concerning the reaction mechanism, it was proposed that the overall partial oxidation is the combination of the total combustion of methane coupled with hydrogen reduction of $\mathrm{CO}_{2}[135]$ :

$$
\begin{gathered}
\mathrm{CH}_{4}+2 \mathrm{O}_{\text {surf }} \longrightarrow \mathrm{CO}_{2}+2 \mathrm{H}_{2} \\
\mathrm{CO}_{2}+\mathrm{H}_{2} \longrightarrow \mathrm{CO}+\mathrm{H}_{2} \mathrm{O} \\
\mathrm{CO}_{2} \longrightarrow \mathrm{CO}+\mathrm{O}_{\text {surf }}
\end{gathered}
$$

When instead of methane, ethane or light alkanes are reacted with $\mathrm{CO}_{2}$ using $\mathrm{Cr}_{2} \mathrm{O}_{3}$-DNPs, then dehydrogenation of ethane and light alkanes takes place (Scheme 20) [136]. The yield of $\mathrm{C}_{2} \mathrm{H}_{4}$ increases along of the oxidation state of chromium oxide present on the DNP catalyst. It was observed that the presence of oxygenated functional groups on the surface of diamond plays a key role in the dehydrogenation by acting as oxygen supplier in the formation of water. Oxygen becomes subsequently replenished by $\mathrm{CO}_{2}$. According to this reaction mechanism, $\mathrm{CO}_{2}$ under the reaction conditions will transfer oxygen atoms to DNPs, becoming converted into $\mathrm{CO}$ [136].

$\mathrm{V}_{2} \mathrm{O}_{5}$ supported on DNPs is also able to promote the reaction of methane and ethane with $\mathrm{CO}_{2}$ but exhibits in general a different reactivity than Ni NPs or $\mathrm{Cr}_{2} \mathrm{O}_{3}$ NPs [137]. In the case of $\mathrm{V}_{2} \mathrm{O}_{5}$-DNPs, the result of the reaction is the corresponding aldehyde, indicating that there is a transfer of an oxygen atom to the alkane (see (6)). Catalytic measurements have shown that formaldehyde yield increases with the increase of the partial pressure of $\mathrm{CO}_{2}$ and with the increase of the space velocity [137]. The later observation was explained as derived from the fact that long residence time of formaldehyde on the catalyst leads to its decomposition. The optimal $\mathrm{V}_{2} \mathrm{O}_{5}$-DNP contains $2 \mathrm{wt} \%$ of $\mathrm{V}_{2} \mathrm{O}_{5}$ loading and the maximum TOF measured was $2.7 \mathrm{molHCHO} \times \mathrm{h}^{-1} \times \mathrm{molV}_{2} \mathrm{O}_{5}{ }^{-1}$ [137]. Similar trends were observed for the formation of acetaldehyde by oxidation of ethane by $\mathrm{CO}_{2}$. As in the related dehydrogenation with $\mathrm{Cr}_{2} \mathrm{O}_{3}$-DNP, it was proposed that the oxygen atoms of $\mathrm{V}_{2} \mathrm{O}_{5}$ and on the surface of DNP are transferred to $\mathrm{C}_{2} \mathrm{H}_{6}$ to form $\mathrm{CH}_{3} \mathrm{CHO}$ and that the role of $\mathrm{CO}_{2}$ is replenishing surface oxygen atoms to DNP:

$$
\mathrm{CH}_{3} \mathrm{CH}_{3}+2 \mathrm{CO}_{2} \underset{\mathrm{V}_{2} \mathrm{O}_{5}-\mathrm{DNP}}{\longrightarrow} \mathrm{CH}_{3} \mathrm{CHO}+2 \mathrm{CO}+\mathrm{H}_{2} \mathrm{O}
$$

The role of $\mathrm{CO}_{2}$ providing oxygen atoms to the surface of DNPs avoids deposition of elemental $\mathrm{C}$ on the catalyst that is the main cause of the lack of selectivity and deactivation of the catalyst. If Ni-DNP or Pd-DNP are used as catalysts for the pyrolysis of ethane or methane, then filamentous carbon nanotubes are formed by decomposition of this hydrocarbon $[138,139]$. As it is usually observed, due to the higher strength of $\mathrm{C}-\mathrm{H}$ bonds, dehydrogenative decomposition of methane requires temperatures higher than those for the case of ethane 


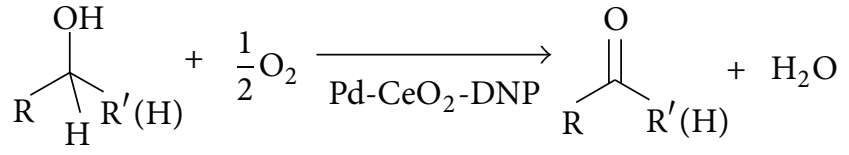

SCHeme 21: Aerobic oxidation of alcohols.

that can be decomposed at temperatures between 400 and $600^{\circ} \mathrm{C}$ in the case Ni-DNP or 500 to $800^{\circ} \mathrm{C}$ in the case of PdDNP. It was observed that temperatures above $650^{\circ} \mathrm{C}$ lead to deactivation of $\mathrm{Ni}-\mathrm{DNP}$ due to the formation of $\mathrm{NiC}_{x}$ phases [138]. In fact, the morphology of the metal NPs changes under the reaction conditions from spherical particles to faceted thin flat particles under operation conditions [138]. Annealing of the resulting thin carbon filaments at $800^{\circ} \mathrm{C}$ for $5 \mathrm{~h}$ under argon also changes the morphology of the carbon filaments to CNTs with high diameters in the range from 80 to $130 \mathrm{~nm}$.

Oxidation of alcohols to carbonyl compounds is a process of large importance in organic synthesis as well as for the preparation of commodities and fine chemicals. A long goal in this area is to develop a general catalyst that can promote selectively alcohol oxidation using molecular oxygen or air. In this regard, it has been reported that Pd NPs combined with $\mathrm{CeO}_{2}$ NPs supported on diamond is able to catalyze this reaction (Scheme 21) [140]. As in other cases, preparation of the material was performed by two consecutive impregnation cycles, first with $\mathrm{Pd}(\mathrm{OAc})_{2}$ and then $\mathrm{Ce}\left(\mathrm{NH}_{4}\right)_{2}\left(\mathrm{NO}_{3}\right)_{6}$, followed by solvent removal and air calcination at $450^{\circ} \mathrm{C}$ for $5 \mathrm{~h}$ [140]. Before using as catalyst, it was necessary to treat the Pd$\mathrm{CeO}_{2}-\mathrm{DNP}$ with a hydrogen stream at $85^{\circ} \mathrm{C}$ for $1 \mathrm{~h}$ to reduce Pd(II) to Pd NPs. In this way, conversions of $95 \%$ of benzyl alcohol to afford $78 \%$ benzaldehyde were achieved [140]. The TOF value of the catalyst was $850 \mathrm{~h}^{-1}$. It was proposed that DNP as support contributes to the catalysis by providing a hydrophobic environment to the active sites avoiding strong water adsorption on the sites. In addition, the lack of porosity of DNP determines that the reaction takes place on a fully accessible external surface. Comparison of the performance of Pd-CeO ${ }_{2}$-DNP with analogous Pd-DNP catalyst lacking $\mathrm{CeO}_{2}$ for the oxidation of 1-phenylethanol shows that the role of $\mathrm{CeO}_{2}$ should be neutralization of the adventitious acid sites on the catalyst surface that are responsible for the lack of selectivity leading to the formation of undesirable methyl benzyl ether and ethyl benzene as secondary products. Other basic metal oxides such as $\mathrm{Y}_{2} \mathrm{O}_{3}$ perform similarly to $\mathrm{CeO}_{2}$, avoiding the acidity introduced by $\mathrm{Pd}$ [140]. Also, comparison of the average particle size for Pd-DNP and $\mathrm{Pd}-\mathrm{CeO}_{2}-\mathrm{DNP}$ shows that an additional role of $\mathrm{CeO}_{2}$ is to favor Pd dispersion reducing the average particle size from 4.7 ( $\mathrm{Pd}-\mathrm{DNP})$ to $3.9 \mathrm{~nm}\left(\mathrm{Pd}-\mathrm{CeO}_{2}\right.$-DNP) [140]:

Fischer-Tropsch synthesis of hydrocarbons is a wellproven technology for the production of fuels from $\mathrm{CO}$ and $\mathrm{H}_{2}$ mixtures of different origins. DNPs have also been used as supports of Co NPs that have high activity for the FischerTropsch synthesis [141]. Two different metal salts, either $\mathrm{Co}\left(\mathrm{NO}_{3}\right)_{2} 6 \mathrm{H}_{2} \mathrm{O}$ or $\mathrm{Co}(\mathrm{OAc})_{2}$, were used in the impregnation of DNPs as cobalt precursors. Impregnation can be carried out either in aqueous solution $\left(\mathrm{Co}\left(\mathrm{NO}_{3}\right)_{2} 6 \mathrm{H}_{2} \mathrm{O}\right)$ or in acetone $\left(\mathrm{Co}(\mathrm{OAc})_{2}\right)$. An interesting aspect of this work has been to show the superior performance of DNPs as support of Co NPs compared to graphite or ACs, even though DNPs have lower surface area than the other two carbon supports. To rationalize this higher activity of DNPs, it was proposed that $\mathrm{sp}^{2}$ carbons exert a negative influence on the Co atoms at the interface, by transferring electron density from the support to the metal, decreasing its catalytic activity [141]. This proposal is again in line with the general fact that for some reactions the inertness of DNP surface can be beneficial for some processes.

Several factors play a key role in the catalytic activity for the Fischer-Tropsch transformation of Co-DNP, such as the reduction temperature in the catalyst pretreatment that influences Co particle size, the reaction temperature that determines the selectivity for methane and $\mathrm{C}_{5+}$ hydrocarbons, and the partial pressure of $\mathrm{H}_{2}$ and $\mathrm{CO}$. All these parameters, including metal precursor salt and Co loading, determine the catalytic activity of the Co-DNP catalyst and the selectivity of the process that, in general, has to be adjusted to optimize the product distribution in $\mathrm{C}_{5+}$ hydrocarbons that can be used as fuels and gasoline alternative. Under optimal conditions, Co-DNP becomes a very stable catalyst, maintaining a steady conversion for one day of continuous flow operation.

Besides being used as supports of metal NPs, DNPs offer other possibilities in catalysis. Due to the high density of surface $\mathrm{OH}$ groups, DNPs can also be used advantageously to anchor covalently some moieties, for instance, by using acyl chlorides or alkoxysilane reagents as reactive functional groups to attach the moiety to the surface [29]. This strategy has, however, still to be further exploited in catalysis for anchoring transition metal complexes as it has been already reported for CNTs and G [142]. In comparison to the last materials, DNPs offering inert surfaces should in principle exhibit a reactivity of the transition metal complex more alike to that observed for homogeneous phase analogues.

\section{Summary and Future Prospects}

In the above sections, I have illustrated the potential that nanostructured allotropic carbon materials offer in catalysis either as carbocatalysts or as supports of active sites. In those cases in which the material can be suspended indefinitely, the system can work similarly to a homogeneous catalyst with the added advantage of being recoverable at the end of the reaction. It has been found that the CNTs and Gs having extended $\pi$ orbitals can interact strongly with substrates and metal NPs, and in this way these carbon supports can influence the catalytic activity by favoring the contact of substrates with the active sites.

Another aspect is that CNTs and G can assist by epitaxial interactions the preferential growth of certain crystallographic facets in the metal NPs while maintaining their small average particle size and influencing their electronic density on the metal NP. These factors can exert strong influence in the catalytic activity exposing the most active metal facets and tuning the electronic density on the metal atoms. 
However, these carbon materials constituted by $\mathrm{sp}^{2}$ atoms may suffer from poor stability when highly reactive intermediates are generated due to the single-layer $\mathrm{G}$ structure or due to the tendency to undergo oxidation and degradation. In contrast, in the other extreme, DNPs conveniently purified from amorphous soot matrix offer an intrinsically robust and inert surface while still allowing anchoring of NPs and stabilization of very small average size particles due to the presence of $-\mathrm{OH}$ nests on the surface. Thus, DNPs are more suited for those reactions in which the role of the support is to provide a high dispersion of the metal NP, without possessing directly any intrinsic catalytic activity.

Considering the availability of new allotropic nanostructured carbon materials and their unique properties derived from well-defined morphologies, high surface area, and predictable interactions, it can be anticipated that their use in catalysis will grow in the near future $[17,21,30]$. Particularly, $\mathrm{G}$ materials can have some advantage over CNTs due to the wider availability and their more convenient preparation and modification [30]. Similarly, the use of DNPs will also grow and will be particularly suited for reactions carried out under harsh conditions and in where highly aggressive and reactive intermediates are generated.

\section{Conflict of Interests}

The author declares that there is no conflict of interests regarding the publication of this paper.

\section{Acknowledgments}

Financial support by the Spanish Ministry of Economy and Competitiveness (Severo Ochoa and CTQ-2012/32315) and Generalitat Valenciana (Prometeo 2012/014) is gratefully acknowledged.

\section{References}

[1] A. E. Aksoylu, M. Madalena, A. Freitas, M. F. R. Pereira, and J. L. Figueiredo, "Effects of different activated carbon supports and support modifications on the properties of Pt/AC catalysts," Carbon, vol. 39, no. 2, pp. 175-185, 2001.

[2] H. Jüntgen, "Activated carbon as catalyst support. A review of new research results," Fuel, vol. 65, no. 10, pp. 1436-1446, 1986.

[3] K. Köhler, R. G. Heidenreich, J. G. E. Krauter, and J. Pietsch, "Highly active palladium/activated carbon catalysts for Heck reactions: correlation of activity, catalyst properties, and $\mathrm{Pd}$ leaching," Chemistry - A European Journal, vol. 8, no. 3, pp. 622631, 2002.

[4] J. Laine, A. Calafat, and M. labady, "Preparation and characterization of activated carbons from coconut shell impregnated with phosphoric acid," Carbon, vol. 27, no. 2, pp. 191-195, 1989.

[5] O. S. Amuda, A. A. Giwa, and I. A. Bello, "Removal of heavy metal from industrial wastewater using modified activated coconut shell carbon," Biochemical Engineering Journal, vol. 36, no. 2, pp. 174-181, 2007.

[6] O. Ioannidou and A. Zabaniotou, "Agricultural residues as precursors for activated carbon production-a review," Renewable and Sustainable Energy Reviews, vol. 11, no. 9, pp. 1966-2005, 2007.
[7] W. K. Lafi, "Production of activated carbon from acorns and olive seeds," Biomass and Bioenergy, vol. 20, no. 1, pp. 57-62, 2001.

[8] A. Zabaniotou, G. Stavropoulos, and V. Skoulou, "Activated carbon from olive kernels in a two-stage process: industrial improvement," Bioresource Technology, vol. 99, no. 2, pp. 320326, 2008.

[9] D. Astruc, F. Lu, and J. R. Aranzaes, "Nanoparticles as recyclable catalysts: the frontier between homogeneous and heterogeneous catalysis," Angewandte Chemie - International Edition, vol. 44, no. 48, pp. 7852-7872, 2005.

[10] G. W. Coates, "Precise control of polyolefin stereochemistry using single-site metal catalysts," Chemical Reviews, vol. 100, no. 4, pp. 1223-1252, 2000.

[11] G. G. Hlatky, "Heterogeneous single-site catalysts for olefin polymerization," Chemical Reviews, vol. 100, no. 4, pp. 13471376, 2000.

[12] J. M. Thomas, R. Raja, and D. W. Lewis, "Single-site heterogeneous catalysts," Angewandte Chemie-International Edition, vol. 44, no. 40, pp. 6456-6482, 2005.

[13] G. Centi and S. Perathoner, "Opportunities and prospects in the chemical recycling of carbon dioxide to fuels," Catalysis Today, vol. 148, no. 3-4, pp. 191-205, 2009.

[14] P. Chawla, V. Chawla, R. Maheshwari, S. A. Saraf, and S. K. Saraf, "Fullerenes: from carbon to nanomedicine," Mini-Reviews in Medicinal Chemistry, vol. 10, no. 8, pp. 662-677, 2010.

[15] R. Schloegl, "Carbon in catalysis," in Advances in Catalysis, B. C. Gates and F. C. Jentoft, Eds., vol. 56, pp. 103-185, 2013.

[16] R. Puskás, A. Sápi, A. Kukovecz, and Z. Kónya, "Comparison of nanoscaled palladium catalysts supported on various carbon allotropes," Topics in Catalysis, vol. 55, no. 11-13, pp. 865-872, 2012.

[17] E. Auer, A. Freund, J. Pietsch, and T. Tacke, "Carbons as supports for industrial precious metal catalysts," Applied Catalysis A: General, vol. 173, no. 2, pp. 259-271, 1998.

[18] M. Kang, Y.-S. Bae, and C.-H. Lee, "Effect of heat treatment of activated carbon supports on the loading and activity of $\mathrm{Pt}$ catalyst," Carbon, vol. 43, no. 7, pp. 1512-1516, 2005.

[19] N. Keller, N. I. Maksimova, V. V. Roddatis et al., "The catalytic use onion-like carbon materials for styrene synthesis by oxidative dehydrogenation ethylbenzene," Angewandte Chemie International Edition, vol. 41, no. 11, pp. 1885-1888, 2002.

[20] L. Tan, B. Wang, and H. Feng, "Comparative studies of graphene oxide and reduced graphene oxide as carbocatalysts for polymerization of 3-aminophenylboronic acid," RSC Advances, vol. 3, no. 8, pp. 2561-2565, 2013.

[21] D. R. Dreyer, H.-P. Jia, and C. W. Bielawski, "Graphene oxide: a convenient carbocatalyst for facilitating oxidation and hydration reactions," Angewandte Chemie, vol. 49, no. 38, pp. 68136816, 2010.

[22] D. R. Dreyer and C. W. Bielawski, "Carbocatalysis: heterogeneous carbons finding utility in synthetic chemistry," Chemical Science, vol. 2, no. 7, pp. 1233-1240, 2011.

[23] J. Pyun, "Graphene oxide as catalyst: application of carbon materials beyond nanotechnology," Angewandte Chemie, vol. 50, no. 1, pp. 46-48, 2011.

[24] C. Su and K. P. Loh, "Carbocatalysts: Graphene oxide and its derivatives," Accounts of Chemical Research, vol. 46, no. 10, pp. 2275-2285, 2013.

[25] D. S. Su, S. Perathoner, and G. Centi, "Nanocarbons for the development of advanced catalysts," Chemical Reviews, vol. 113, no. 8, pp. 5782-5816, 2013. 
[26] M. S. Dresselhaus and M. Terrones, "Carbon-based nanomaterials from a historical perspective," Proceedings of the IEEE, vol. 101, no. 7, pp. 1522-1535, 2013.

[27] M. Endo, T. Hayashi, Y.-A. Kim, M. Terrones, and M. S. Dresselhaus, "History and structure in carbon nanotube," Chimica Oggi-Chemistry Today, vol. 23, no. 2, pp. 29-32, 2005.

[28] V. Y. Dolmatov, "Detonation synthesis ultradispersed diamonds: properties and applications," Russian Chemical Reviews, vol. 70, no. 7, pp. 607-626, 2001.

[29] R. Martín, P. C. Heydorn, M. Alvaro, and H. Garcia, "General strategy for high-density covalent functionalization of diamond nanoparticles using fenton chemistry," Chemistry of Materials, vol. 21, no. 19, pp. 4505-4514, 2009.

[30] C. Huang, C. Li, and G. Shi, “Graphene based catalysts," Energy and Environmental Science, vol. 5, no. 10, pp. 8848-8868, 2012.

[31] D. R. Dreyer, K. A. Jarvis, P. J. Ferreira, and C. W. Bielawski, "Graphite oxide as a carbocatalyst for the preparation of fullerene-reinforced polyester and polyamide nanocomposites," Polymer Chemistry, vol. 3, no. 3, pp. 757-766, 2012.

[32] M. Boronat and A. Corma, "Molecular approaches to catalysis: naked gold nanoparticles as quasi-molecular catalysts for green processes," Journal of Catalysis, vol. 284, no. 2, pp. 138-147, 2011.

[33] A. Corma, H. Garcia, and A. Leyva, "Catalytic activity of palladium supported on single wall carbon nanotubes compared to palladium supported on activated carbon: study of the Heck and Suzuki couplings, aerobic alcohol oxidation and selective hydrogenation," Journal of Molecular Catalysis A: Chemical, vol. 230, no. 1-2, pp. 97-105, 2005.

[34] E. Flahaut, A. Govindaraj, A. Peigney, C. Laurent, A. Rousset, and C. N. R. Rao, "Synthesis of single-walled carbon nanotubes using binary (Fe, $\mathrm{Co}, \mathrm{Ni}$ ) alloy nanoparticles prepared in situ by the reduction of oxide solid solutions," Chemical Physics Letters, vol. 300, no. 1-2, pp. 236-242, 1999.

[35] A. Dhakshinamoorthy, A. Primo, P. Concepcion, M. Alvaro, and H. Garcia, "Doped graphene as a metal-free carbocatalyst for the selective aerobic oxidation of benzylic hydrocarbons, cyclooctane and styrene," Chemistry, vol. 19, no. 23, pp. 75477554, 2013.

[36] T. W. Ebbesen and P. M. Ajayan, "Large-scale synthesis of carbon nanotubes," Nature, vol. 358, no. 6383, pp. 220-222, 1992.

[37] P. M. Ajayan, "Nanotubes from Carbon," Chemical Reviews, vol. 99, no. 7, pp. 1787-1799, 1999.

[38] D. S. Bethune, C. H. Kiang, M. S. de Vries et al., "Cobaltcatalysed growth of carbon nanotubes with single-atomic-layer walls," Nature, vol. 363, no. 6430, pp. 605-607, 1993.

[39] C. Aprile, R. Martin, M. Alvaro, J. C. Scaiano, and H. Garcia, "Near-infrared emission quantum yield of soluble short singlewalled carbon nanotubes," Chemphyschem, vol. 10, no. 8, pp. 1305-1310, 2009.

[40] R. Martín, M. Álvaro, and H. García, "Photoresponsive covalently-functionalized short single wall carbon nanotubes," Current Organic Chemistry, vol. 15, no. 8, pp. 1106-1120, 2011.

[41] M. F. Islam, E. Rojas, D. M. Bergey, A. T. Johnson, and A. G. Yodh, "High weight fraction surfactant solubilization of singlewall carbon nanotubes in water," Nano Letters, vol. 3, no. 2, pp. 269-273, 2003.

[42] M. Zheng, A. Jagota, E. D. Semke et al., "DNA-assisted dispersion and separation of carbon nanotubes," Nature Materials, vol. 2, no. 5, pp. 338-342, 2003.

[43] X. Liu, B. Frank, W. Zhang, T. P. Cotter, R. Schlögl, and D. S. $\mathrm{Su}$, "Carbon-catalyzed oxidative dehydrogenation of $n$-butane: selective site formation during $\mathrm{sp}^{3}$-to-sp $\mathrm{p}^{2}$ lattice rearrangement," Angewandte Chemie, vol. 50, no. 14, pp. 3318-3322, 2011.

[44] W. Qi, W. Liu, B. Zhang, X. Gu, X. Guo, and D. Su, "Oxidative dehydrogenation on nanocarbon: identification and quantification of active sites by chemical titration," Angewandte Chemie, vol. 52, no. 52, pp. 14224-14228, 2013.

[45] J. Zhang, X. Liu, R. Blume, A. Zhang, R. Schlögl, and S. S. Dang, "Surface-modified carbon nanotubes catalyze oxidative dehydrogenation of n-butane," Science, vol. 322, no. 5898, pp. 73-77, 2008.

[46] X. Liu, D. S. Su, and R. Schlögl, "Oxidative dehydrogenation of 1-butene to butadiene over carbon nanotube catalysts," Carbon, vol. 46, no. 3, pp. 547-549, 2008.

[47] A. Dhakshinamoorthy, S. Navalon, M. Alvaro, and H. Garcia, "Metal nanoparticles as heterogeneous fenton catalysts," ChemSusChem, vol. 5, no. 1, pp. 46-64, 2012.

[48] S. Navalon, A. Dhakshinamoorthy, M. Alvaro, and H. Garcia, "Heterogeneous Fenton catalysts based on activated carbon and related materials," ChemSusChem, vol. 4, no. 12, pp. 1712-1730, 2011.

[49] Z. H. Kang, E. B. Wang, B. D. Mao et al., "Heterogeneous hydroxylation catalyzed by multi-walled carbon nanotubes at low temperature," Applied Catalysis A: General, vol. 299, no. 1-2, pp. 212-217, 2006.

[50] S. Song, H. Yang, R. Rao, H. Liu, and A. Zhang, "Defects of multi-walled carbon nanotubes as active sites for benzene hydroxylation to phenol in the presence of $\mathrm{H}_{2} \mathrm{O}_{2}$," Catalysis Communications, vol. 11, no. 8, pp. 783-787, 2010.

[51] H. Zhang, X. Pan, X. Han et al., "Enhancing chemical reactions in a confined hydrophobic environment: an NMR study of benzene hydroxylation in carbon nanotubes," Chemical Science, vol. 4, no. 3, pp. 1075-1078, 2013.

[52] H. Sun, S. Liu, G. Zhou, H. M. Ang, M. O. Tadé, and S. Wang, "Reduced graphene oxide for catalytic oxidation of aqueous organic pollutants," ACS Applied Materials and Interfaces, vol. 4, no. 10, pp. 5466-5471, 2012.

[53] C. Chen, J. Zhang, B. Zhang, C. Yu, F. Peng, and D. Su, "Revealing the enhanced catalytic activity of nitrogen-doped carbon nanotubes for oxidative dehydrogenation of propane," Chemical Communications, vol. 49, no. 74, pp. 8151-8153, 2013.

[54] B. Frank, J. Zhang, R. Blume, R. Schlögl, and D. S. Su, "Heteroatoms increase the selectivity in oxidative dehydrogenation reactions on nanocarbons," Angewandte Chemie-International Edition, vol. 48, no. 37, pp. 6913-6917, 2009.

[55] J. Luo, H. Yu, H. Wang, H. Wang, and F. Peng, "Aerobic oxidation of benzyl alcohol to benzaldehyde catalyzed by carbon nanotubes without any promoter," Chemical Engineering Journal, vol. 240, pp. 434-442, 2014.

[56] J. Luo, F. Peng, H. Wang, and H. Yu, "Enhancing the catalytic activity of carbon nanotubes by nitrogen doping in the selective liquid phase oxidation of benzyl alcohol," Catalysis Communications, vol. 39, pp. 44-49, 2013.

[57] Y. Cao, X. Luo, H. Yu, F. Peng, H. Wang, and G. Ning, "Sp2and sp3-hybridized carbon materials as catalysts for aerobic oxidation of cyclohexane," Catalysis Science and Technology, vol. 3, no. 10, pp. 2654-2660, 2013.

[58] Z.-Z. Fang, X.-D. Kang, P. Wang, and H.-M. Cheng, "Improved reversible dehydrogenation of lithium borohydride by milling with as-prepared single-walled carbon nanotubes," Journal of Physical Chemistry C, vol. 112, no. 43, pp. 17023-17029, 2008. 
[59] P.-J. Wang, Z.-Z. Fang, L.-P. Ma, X.-D. Kang, and P. Wang, "Effect of carbon addition on hydrogen storage behaviors of $\mathrm{Li}$ Mg-B-H system," International Journal of Hydrogen Energy, vol. 35, no. 7, pp. 3072-3075, 2010.

[60] X. B. Yu, Z. Wu, Q. R. Chen, Z. L. Li, B. C. Weng, and T. S. Huang, "Improved hydrogen storage properties of LiBH4 destabilized by carbon," Applied Physics Letters, vol. 90, no. 3, Article ID 034106, 2007.

[61] Y. Zhang, W.-S. Zhang, A.-Q. Wang et al., " $\mathrm{LiBH}_{4}$ nanoparticles supported by disordered mesoporous carbon: hydrogen storage performances and destabilization mechanisms," International Journal of Hydrogen Energy, vol. 32, no. 16, pp. 3976-3980, 2007.

[62] P. Serp, M. Corrias, and P. Kalck, "Carbon nanotubes and nanofibers in catalysis," Applied Catalysis A: General, vol. 253, no. 2, pp. 337-358, 2003.

[63] J.-P. Tessonnier, L. Pesant, G. Ehret, M. J. Ledoux, and C. PhamHuu, "Pd nanoparticles introduced inside multi-walled carbon nanotubes for selective hydrogenation of cinnamaldehyde into hydrocinnamaldehyde," Applied Catalysis A: General, vol. 288, no. 1-2, pp. 203-210, 2005.

[64] X. R. Ye, Y. Lin, and C. M. Wai, "Decorating catalytic palladium nanoparticles on carbon nanotubes in supercritical carbon dioxide," Chemical Communications, vol. 9, no. 5, pp. 642-643, 2003.

[65] G.-Y. Gao, D.-J. Guo, and H.-L. Li, "Electrocatalytic oxidation of formaldehyde on palladium nanoparticles supported on multiwalled carbon nanotubes," Journal of Power Sources, vol. 162, no. 2, pp. 1094-1098, 2006.

[66] B. Yoon and C. M. Wai, "Microemulsion-templated synthesis of carbon nanotube-supported Pd and Rh nanoparticles for catalytic applications," Journal of the American Chemical Society, vol. 127, no. 49, pp. 17174-17175, 2005.

[67] F. Ding, P. Larsson, J. A. Larsson et al., "The importance of strong carbon-metal adhesion for catalytic nucleation of singlewalled carbon nanotubes," Nano Letters, vol. 8, no. 2, pp. 463468, 2008.

[68] L. Alves, B. Ballesteros, M. Boronat et al., "Synthesis and stabilization of subnanometric gold oxide nanoparticles on multiwalled carbon nanotubes and their catalytic activity," Journal of the American Chemical Society, vol. 133, no. 26, pp. 10251-10261, 2011.

[69] A. Abad, A. Corma, and H. García, "Catalyst parameters determining activity and selectivity of supported gold nanoparticles for the aerobic oxidation of alcohols: The molecular reaction mechanism," Chemistry - A European Journal, vol. 14, no. 1, pp. 212-222, 2008.

[70] A. Corma, P. Concepción, M. Boronat et al., "Exceptional oxidation activity with size-controlled supported gold clusters of low atomicity," Nature Chemistry, vol. 5, no. 9, pp. 775-781, 2013.

[71] C. Baleizão, B. Gigante, H. García, and A. Corma, "Chiral vanadyl salen complex anchored on supports as recoverable catalysts for the enantioselective cyanosilylation of aldehydes. Comparison among silica, single wall carbon nanotube, activated carbon and imidazolium ion as support," Tetrahedron, vol. 60, no. 46, pp. 10461-10468, 2004.

[72] C. Baleizão, B. Gigante, H. Garcia, and A. Corma, "Vanadyl salen complexes covalently anchored to single-wall carbon nanotubes as heterogeneous catalysts for the cyanosilylation of aldehydes," Journal of Catalysis, vol. 221, no. 1, pp. 77-84, 2004.
[73] D. Tasis, N. Tagmatarchis, A. Bianco, and M. Prato, "Chemistry of carbon nanotubes," Chemical Reviews, vol. 106, no. 3, pp. 1105-1136, 2006.

[74] J. L. Hutchison, N. A. Kiselev, E. P. Krinichnaya et al., "Doublewalled carbon nanotubes fabricated by a hydrogen arc discharge method," Carbon, vol. 39, no. 5, pp. 761-770, 2001.

[75] J. Kong, A. M. Cassell, and H. Dai, "Chemical vapor deposition of methane for single-walled carbon nanotubes," Chemical Physics Letters, vol. 292, no. 4-6, pp. 567-574, 1998.

[76] S. Stankovich, D. A. Dikin, R. D. Piner et al., "Synthesis of graphene-based nanosheets via chemical reduction of exfoliated graphite oxide," Carbon, vol. 45, no. 7, pp. 1558-1565, 2007.

[77] W. S. Hummers Jr. and R. E. Offeman, "Preparation of graphitic oxide," Journal of the American Chemical Society, vol. 80, no. 6, p. 1339, 1958.

[78] A. Primo, P. Atienzar, E. Sanchez, J. M. Delgado, and H. García, "From biomass wastes to large-area, high-quality, N-doped graphene: catalyst-free carbonization of chitosan coatings on arbitrary substrates," Chemical Communications, vol. 48, no. 74, pp. 9254-9256, 2012.

[79] P. Atienzar, A. Primo, C. Lavorato, R. Molinari, and H. García, "Preparation of graphene quantum dots from pyrolyzed alginate," Langmuir, vol. 29, no. 20, pp. 6141-6146, 2013.

[80] A. Primo, A. Forneli, A. Corma, and H. García, "From biomass wastes to highly efficient $\mathrm{CO}_{2}$ adsorbents: graphitisation of chitosan and alginate biopolymers," ChemSusChem, vol. 5, no. 11, pp. 2207-2214, 2012.

[81] C. Su, M. Acik, K. Takai et al., "Probing the catalytic activity of porous graphene oxide and the origin of this behaviour," Nature Communications, vol. 3, article 2315, 8 pages, 2012.

[82] D. R. Dreyer, S. Park, C. W. Bielawski, and R. S. Ruoff, "The chemistry of graphene oxide," Chemical Society Reviews, vol. 39, no. 1, pp. 228-240, 2010.

[83] N. Wu, X. She, D. Yang, X. Wu, F. Su, and Y. Chen, "Synthesis of network reduced graphene oxide in polystyrene matrix by a two-step reduction method for superior conductivity of the composite," Journal of Materials Chemistry, vol. 22, no. 33, pp. 17254-17261, 2012.

[84] A. Dhakshinamoorthy, M. Alvaro, P. Concepción, V. Fornés, and H. Garcia, "Graphene oxide as an acid catalyst for the room temperature ring opening of epoxides," Chemical Communications, vol. 48, no. 44, pp. 5443-5445, 2012.

[85] A. Dhakshinamoorthy, M. Alvaro, M. Puche, V. Fornes, and H. Garcia, "Graphene oxide as catalyst for the acetalizacion of aldehydes at room temperature," Chem CatChem, vol. 4, no. 12, pp. 2026-2030, 2012.

[86] L. Qu, Y. Liu, J.-B. Baek, and L. Dai, "Nitrogen-doped graphene as efficient metal-free electrocatalyst for oxygen reduction in fuel cells," ACS Nano, vol. 4, no. 3, pp. 1321-1326, 2010.

[87] A. Corma and H. Garcia, "Supported gold nanoparticles as catalysts for organic reactions," Chemical Society Reviews, vol. 37, no. 9, pp. 2096-2126, 2008.

[88] W. Peng, S. Liu, H. Sun, Y. Yao, L. Zhi, and S. Wang, "Synthesis of porous reduced graphene oxide as metal-free carbon for adsorption and catalytic oxidation of organics in water," Journal of Materials Chemistry A, vol. 1, pp. 5854-5859, 2013.

[89] Y. Gao, D. Ma, C. Wang, J. Guan, and X. Bao, "Reduced graphene oxide as a catalyst for hydrogenation of nitrobenzene at room temperature," Chemical Communications, vol. 47, no. 8 , pp. 2432-2434, 2011. 
[90] X.-K. Kong, Z.-Y. Sun, M. Chen, C.-L. Chen, and Q.-W. Chen, "Metal-free catalytic reduction of 4-nitrophenol to 4aminophenol by N-doped graphene," Energy and Environmental Science, vol. 6, no. 11, pp. 3260-3266, 2013.

[91] X. K. Kong, Q. W. Chen, and Z. Y. Lun, "Probing the influence of different oxygenated groups on graphene oxide's catalytic performance," Journal of Materials Chemistry A, vol. 2, no. 3, pp. 610-613, 2014.

[92] V. Schwartz, W. Fu, Y.-T. Tsai et al., "Oxygen-functionalized few-layer graphene sheets as active catalysts for oxidative dehydrogenation reactions," ChemSusChem, vol. 6, no. 5, pp. 840-846, 2013.

[93] A. Corma and H. Garcia, "Lewis acids: from conventional homogeneous to green homogeneous and heterogeneous catalysis," Chemical Reviews, vol. 103, no. 11, pp. 4307-4366, 2003.

[94] L.-M. Liu, R. Car, A. Selloni, D. M. Dabbs, I. A. Aksay, and R. A. Yetter, "Enhanced thermal decomposition of nitromethane on functionalized graphene sheets: $\mathrm{Ab}$ initio molecular dynamics simulations," Journal of the American Chemical Society, vol. 134, no. 46, pp. 19011-19016, 2012.

[95] J. L. Sabourin, D. M. Dabbs, R. A. Yetter, F. L. Dryer, and I. A. Aksay, "Functionalized graphene sheet colloids for enhanced fuel/propellant combustion," ACS Nano, vol. 3, no. 12, pp. 39453954, 2009.

[96] G. Eda, G. Fanchini, and M. Chhowalla, "Large-area ultrathin films of reduced graphene oxide as a transparent and flexible electronic material," Nature Nanotechnology, vol. 3, no. 5, pp. 270-274, 2008

[97] G. Blanita and M. D. Lazar, "Review of graphene-supported metal nanoparticles as new and efficient heterogeneous catalysts," Micro and Nanosystems, vol. 5, no. 2, pp. 138-146, 2013.

[98] M. Ding, Y. Tang, and A. Star, "Understanding interfaces in metal-graphitic hybrid nanostructures," Journal of Physical Chemistry Letters, vol. 4, no. 1, pp. 147-160, 2013.

[99] S. Sharma, A. Ganguly, P. Papakonstantinou et al., "Rapid microwave synthesis of CO tolerant Reduced graphene oxidesupported platinum electrocatalysts for oxidation of methanol," Journal of Physical Chemistry C, vol. 114, no. 45, pp. 1945919466, 2010.

[100] K. Jasuja, J. Linn, S. Melton, and V. Berry, "Microwave-reduced uncapped metal nanoparticles on graphene: tuning catalytic, electrical, and raman properties," Journal of Physical Chemistry Letters, vol. 1, no. 12, pp. 1853-1860, 2010.

[101] R. Nie, J. Wang, L. Wang, Y. Qin, P. Chen, and Z. Hou, "Platinum supported on reduced graphene oxide as a catalyst for hydrogenation of nitroarenes," Carbon, vol. 50, no. 2, pp. 586-596, 2012.

[102] G. M. Scheuermann, L. Rumi, P. Steurer, W. Bannwarth, and R. Mülhaupt, "Palladium nanoparticles on graphite oxide and its functionalized graphene derivatives as highly active catalysts for the Suzuki-Miyaura coupling reaction," Journal of the American Chemical Society, vol. 131, no. 23, pp. 8262-8270, 2009.

[103] D.-H. Lim and J. Wilcox, "Mechanisms of the oxygen reduction reaction on defective graphene-supported Pt nanoparticles from first-principles," Journal of Physical Chemistry C, vol. 116, no. 5, pp. 3653-3660, 2012.

[104] F. H. Yang, A. J. Lachawiec Jr., and R. T. Yang, "Adsorption of spillover hydrogen atoms on single-wall carbon nanotubes," Journal of Physical Chemistry B, vol. 110, no. 12, pp. 6236-6244, 2006.
[105] N. Shang, P. Papakonstantinou, P. Wang, and S. R. P. Silva, "Platinum integrated graphene for methanol fuel cells," Journal of Physical Chemistry C, vol. 114, no. 37, pp. 15837-15841, 2010.

[106] C. Xu, X. Wang, and J. Zhu, "Graphene-metal particle nanocomposites," Journal of Physical Chemistry C, vol. 112, no. 50, pp. 19841-19845, 2008.

[107] L. Dong, R. R. S. Gari, Z. Li, M. M. Craig, and S. Hou, "Graphene-supported platinum and platinum-ruthenium nanoparticles with high electrocatalytic activity for methanol and ethanol oxidation," Carbon, vol. 48, no. 3, pp. 781-787, 2010.

[108] Y. Li, W. Gao, L. Ci, C. Wang, and P. M. Ajayan, "Catalytic performance of Pt nanoparticles on reduced graphene oxide for methanol electro-oxidation," Carbon, vol. 48, no. 4, pp. 1124$1130,2010$.

[109] C. Li and G. Shi, "Three-dimensional graphene architectures," Nanoscale, vol. 4, no. 18, pp. 5549-5563, 2012.

[110] J. Peng, W. Gao, B. K. Gupta et al., "Graphene quantum dots derived from carbon fibers," Nano Letters, vol. 12, no. 2, pp. 844849, 2012.

[111] G. He, Y. Song, K. Liu, A. Walter, S. Chen, and S. Chen, "Oxygen reduction catalyzed by platinum nanoparticles supported on graphene quantum dots," ACS Catalysis, vol. 3, no. 5, pp. 831838, 2013.

[112] Y. Shao, J. Wang, H. Wu, J. Liu, I. A. Aksay, and Y. Lin, "Graphene based electrochemical sensors and biosensors: a review," Electroanalysis, vol. 22, no. 10, pp. 1027-1036, 2010.

[113] D. A. C. Brownson, D. K. Kampouris, and C. E. Banks, "Graphene electrochemistry: fundamental concepts through to prominent applications," Chemical Society Reviews, vol. 41, no. 21, pp. 6944-6976, 2012.

[114] F. Cheng and J. Chen, "Metal-air batteries: from oxygen reduction electrochemistry to cathode catalysts," Chemical Society Reviews, vol. 41, no. 6, pp. 2172-2192, 2012.

[115] V. Georgakilas, M. Otyepka, A. B. Bourlinos et al., "Functionalization of graphene: covalent and non-covalent approaches, derivatives and applications," Chemical Reviews, vol. 112, no. 11, pp. 6156-6214, 2012.

[116] S. Navalon, M. de Miguel, R. Martin, M. Alvaro, and H. Garcia, "Enhancement of the catalytic activity of supported gold nanoparticles for the fenton reaction by light," Journal of the American Chemical Society, vol. 133, no. 7, pp. 2218-2226, 2011.

[117] S. Navalon, R. Martin, M. Alvaro, and H. Garcia, "Sunlightassisted fenton reaction catalyzed by gold supported on diamond nanoparticles as pretreatment for biological degradation of aqueous phenol solutions," ChemSusChem, vol. 4, no. 5, pp. 650-657, 2011.

[118] R. Martín, M. Álvaro, J. R. Herance, and H. García, "Fentontreated functionalized diamond nanoparticles as gene delivery system," ACS Nano, vol. 4, no. 1, pp. 65-74, 2010.

[119] S. Navalon, R. Martin, M. Alvaro, and H. Garcia, "Gold on diamond nanoparticles as a highly efficient fenton catalyst," Angewandte Chemie, vol. 49, no. 45, pp. 8403-8407, 2010.

[120] J. Feng, X. Hu, and P. L. Yue, "Effect of initial solution $\mathrm{pH}$ on the degradation of Orange II using clay-based Fe nanocomposites as heterogeneous photo-Fenton catalyst," Water Research, vol. 40, no. 4, pp. 641-646, 2006.

[121] M. B. Kasiri, H. Aleboyeh, and A. Aleboyeh, "Degradation of acid blue 74 using Fe-ZSM5 zeolite as a heterogeneous photoFenton catalyst," Applied Catalysis B: Environmental, vol. 84, no. 1-2, pp. 9-15, 2008. 
[122] P. Wardman and L. P. Candeias, "Fenton chemistry: an introduction," Radiation Research, vol. 145, no. 5, pp. 523-531, 1996.

[123] C. Aliaga, D. R. Stuart, A. Aspée, and J. C. Scaiano, "Solvent effects on hydrogen abstraction reactions from lactones with antioxidant properties," Organic Letters, vol. 7, no. 17, pp. 36653668, 2005.

[124] A. Dhakshinamoorthy, S. Navalon, D. Sempere, M. Alvaro, and H. Garcia, "Aerobic oxidation of thiols catalyzed by copper nanoparticles supported on diamond nanoparticles," ChemCatChem, vol. 5, no. 1, pp. 241-246, 2013.

[125] A. Dhakshinamoorthy, S. Navalon, D. Sempere, M. Alvaro, and H. García, "Reduction of alkenes catalyzed by copper nanoparticles supported on diamond nanoparticles," Chemical Communications, vol. 49, no. 23, pp. 2359-2361, 2013.

[126] Y. Wang, Z. Xiao, and L. Wu, "Metal-nanoparticles supported on solid as heterogeneous catalysts," Current Organic Chemistry, vol. 17, no. 12, pp. 1325-1333, 2013.

[127] L. Huang, H. Wang, J. Chen et al., "Synthesis, morphology control, and properties of porous metal-organic coordination polymers," Microporous and Mesoporous Materials, vol. 58, no. 2, pp. 105-114, 2003.

[128] C. Z.-J. Lin, S. S.-Y. Chui, S. M.-F. Lo et al., "Physical stability vs. chemical lability in microporous metal coordination polymers: a comparison of $[\mathrm{Cu}(\mathrm{OH})(\mathrm{INA})]_{n}$ and $\left[\mathrm{Cu}(\mathrm{INA})_{2}\right]_{n}$ : INA = 1,4- $\left(\mathrm{NC}_{5} \mathrm{H}_{4} \mathrm{CO}_{2}\right)$," Chemical Communications, no. 15, pp. 16421643, 2002.

[129] T. M. Reineke, M. Eddaoudi, M. O'Keeffe, and O. M. Yaghi, "A microporous lanthanide-organic framework," Angewandte Chemie International Edition, vol. 38, pp. 2590-2594, 1999.

[130] J. Lee, O. K. Farha, J. Roberts, K. A. Scheidt, S. T. Nguyen, and J. T. Hupp, "Metal-organic framework materials as catalysts," Chemical Society Reviews, vol. 38, no. 5, pp. 1450-1459, 2009.

[131] A. Dhakshinamoorthy, M. Alvaro, and H. Garcia, "Metalorganic frameworks as heterogeneous catalysts for oxidation reactions," Catalysis Science and Technology, vol. 1, no. 6, pp. 856-867, 2011.

[132] A. Dhakshinamoorthy, M. Alvaro, and H. Garcia, "Aerobic oxidation of styrenes catalyzed by an iron metal organic framework," ACS Catalysis, vol. 1, no. 8, pp. 836-840, 2011.

[133] A. Dhakshinamoorthy, M. Alvaro, and H. García, "Aerobic oxidation of thiols to disulfides using iron metal-organic frameworks as solid redox catalysts," Chemical Communications, vol. 46, no. 35, pp. 6476-6478, 2010.

[134] K. Nakagawa, H. Nishimoto, Y. Enoki et al., "Oxidized diamond supported $\mathrm{Ni}$ catalyst for synthesis gas formation from methane," Chemistry Letters, no. 5, pp. 460-461, 2001.

[135] H.-A. Nishimoto, K. Nakagawa, N.-O. Ikenaga, M. NishitaniGamo, T. Ando, and T. Suzuki, "Partial oxidation of methane to synthesis gas over oxidized diamond catalysts," Applied Catalysis A: General, vol. 264, no. 1, pp. 65-72, 2004.

[136] K. Nakagawa, C. Kajita, N.-O. Ikenaga et al., "The role of chemisorbed oxygen on diamond surfaces for the dehydrogenation of ethane in the presence of carbon dioxide," Journal of Physical Chemistry B, vol. 107, no. 17, pp. 4048-4056, 2003.

[137] K. Okumura, K. Nakagawa, T. Shimamura et al., "Direct formation of acetaldehyde from ethane using carbon dioxide as a novel oxidant over oxidized diamond-supported catalysts," The Journal of Physical Chemistry B, vol. 107, no. 48, pp. 1341913424, 2003.

[138] N.-O. Higashi, H.-A. Ichi-oka, T. Miyake, and T. Suzuki, "Growth mechanisms of carbon nanofilaments on Ni-loaded diamond catalyst," Diamond and Related Materials, vol. 17, no. 3, pp. 283-293, 2008.

[139] N.-O. Higashi, N.-O. Ikenaga, T. Miyake, and T. Suzuki, "Carbon nanotube formation on $\mathrm{Ni}$ - or Pd-loaded diamond catalysts," Diamond and Related Materials, vol. 14, no. 3-7, pp. 820-824, 2005.

[140] T. Yasu-eda, R. Se-ike, N.-O. Ikenaga, T. Miyake, and T. Suzuki, "Palladium-loaded oxidized diamond catalysis for the selective oxidation of alcohols," Journal of Molecular Catalysis A: Chemical, vol. 306, no. 1-2, pp. 136-142, 2009.

[141] T.-O. Honsho, T. Kitano, T. Miyake, and T. Suzuki, "FischerTropsch synthesis over Co-loaded oxidized diamond catalyst," Fuel, vol. 94, pp. 170-177, 2012.

[142] P. V. Kamat, "Graphene-based nanoarchitectures: anchoring semiconductor and metal nanoparticles on a two-dimensional carbon support," Journal of Physical Chemistry Letters, vol. 1, no. 2, pp. 520-527, 2010. 

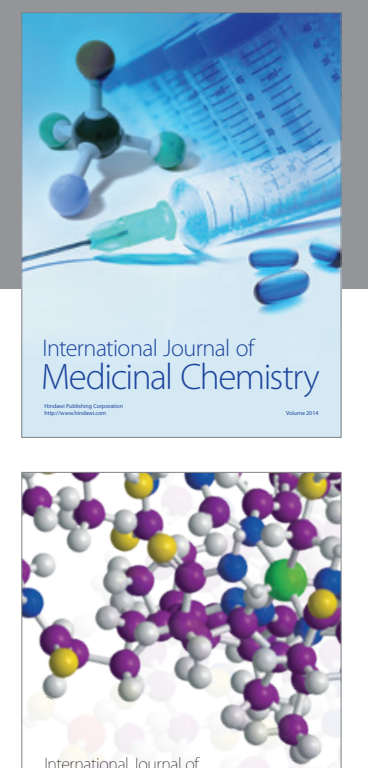

\section{Carbohydrate} Chemistry

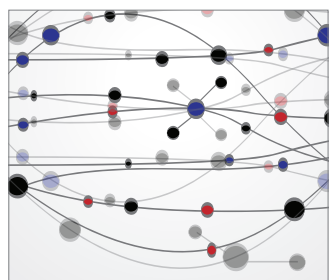

The Scientific World Journal
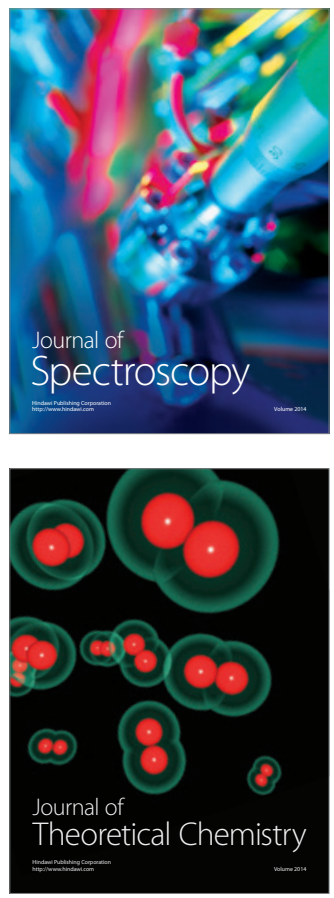
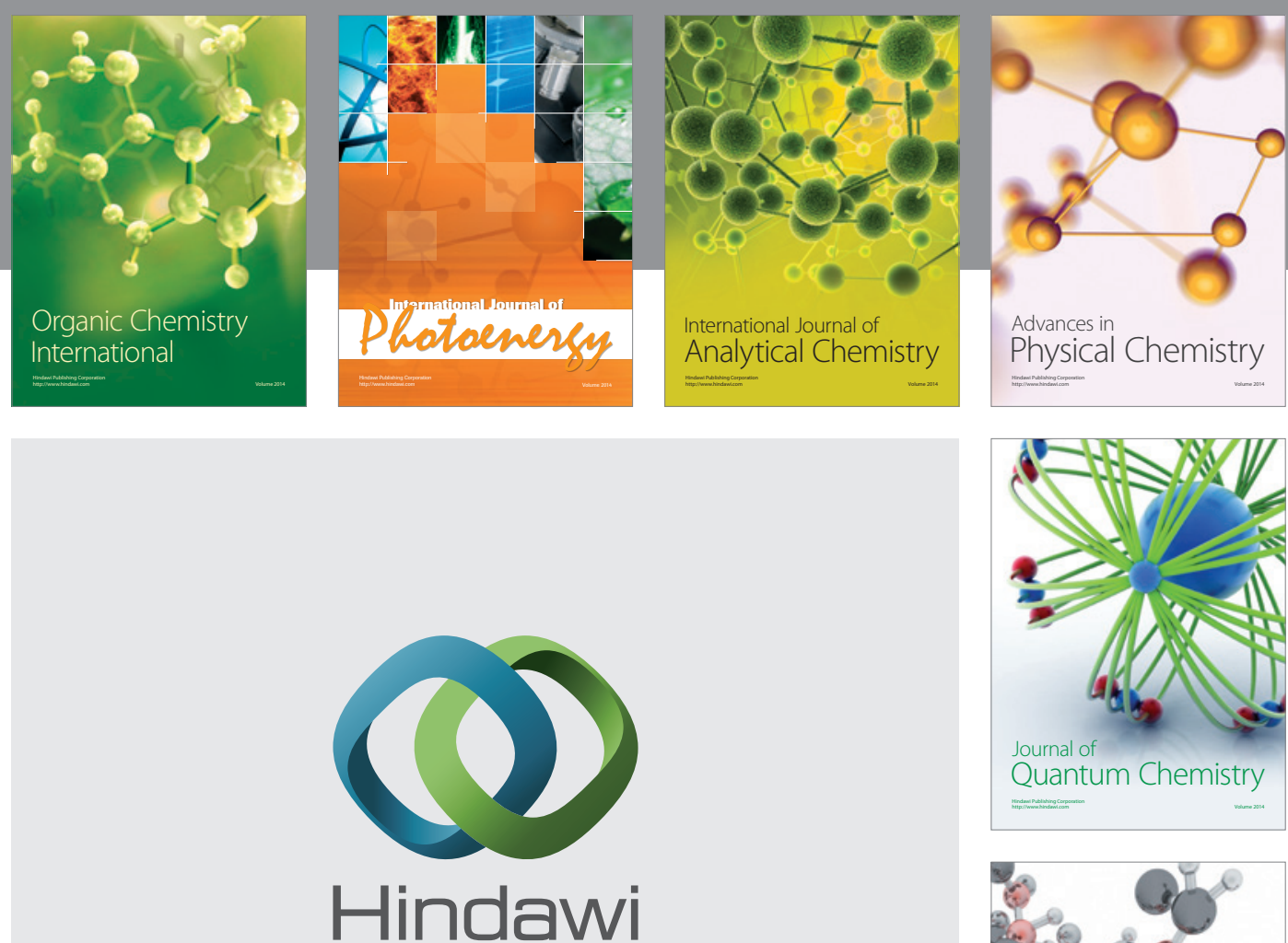

Submit your manuscripts at

http://www.hindawi.com

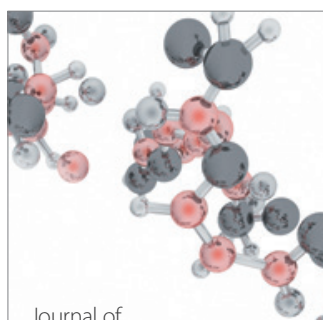

Analytical Methods

in Chemistry

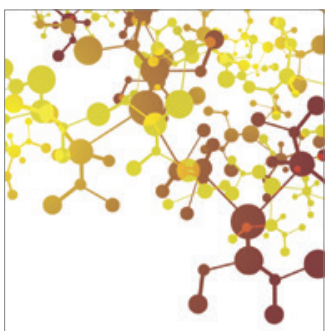

Journal of

Applied Chemistry

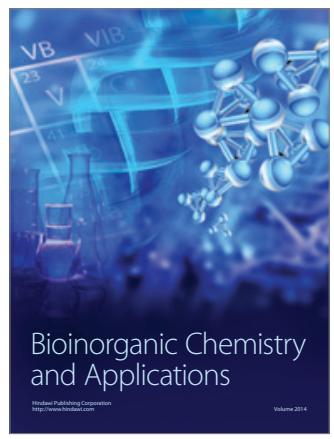

Inorganic Chemistry
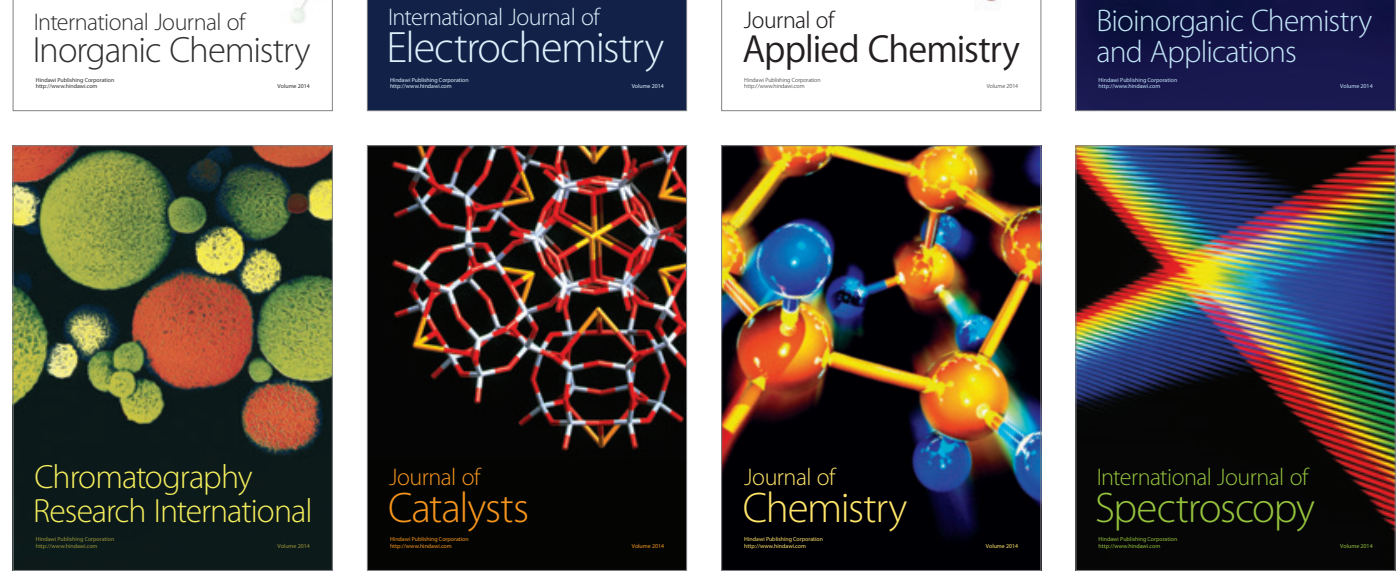